\title{
Fair and efficient student placement with couples
}

\author{
Bettina Klaus • Flip Klijn
}

Accepted: 6 October 2006 / Published online: 30 January 2007

(C) Springer-Verlag 2007

\begin{abstract}
We study situations of allocating positions to students based on priorities. An example is the assignment of medical students to hospital residencies on the basis of entrance exams. For markets without couples, e.g., for undergraduate student placement, acyclicity is a necessary and sufficient condition for the existence of a fair and efficient placement mechanism (Ergin in Econometrica 70:2489-2497, 2002). We show that in the presence of couples acyclicity is still necessary, but not sufficient. A second necessary condition is priority-togetherness of couples. A priority structure that satisfies both necessary conditions is called $p t$-acyclic.

For student placement problems where all quotas are equal to one we characterize $p t$-acyclicity and show that it is a sufficient condition for the existence of a fair and efficient placement mechanism. If in addition to $p t$-acyclicity we require reallocation- and vacancy-fairness for couples, the so-called dictatorbidictator placement mechanism is the unique fair and efficient placement mechanism.

Finally, for general student placement problems, we show that $p t$-acyclicity may not be sufficient for the existence of a fair and efficient placement mechanism. We identify a sufficient condition such that the so-called sequential placement mechanism produces a fair and efficient allocation.
\end{abstract}

\footnotetext{
B. Klaus $(\bowtie)$

Department of Economics, Maastricht University, P.O. Box 616, 6200 MD Maastricht, The Netherlands e-mail: B.Klaus@algec.unimaas.nl

F. Klijn

Institut d'Anàlisi Econòmica (CSIC),

Campus UAB, 08193 Bellaterra (Barcelona), Spain

e-mail: Flip.Klijn@uab.es
} 
Keywords Student placement - Fairness - Efficiency - Couples · Acyclic priority structure

JEL Classification D61 $\cdot \mathrm{D} 63 \cdot \mathrm{D} 70 \cdot \mathrm{C} 78$

\section{Introduction}

We consider so-called house allocation or student placement problems. A student placement problem is determined by a set of position types, the number of available positions of each type (the quota), and the students' strict preferences over position types (e.g., a position type could be a house or a position at a university or firm) and remaining unassigned. A (student) placement mechanism assigns to any given student placement problem an allocation of the position types to the students such that every student receives at most one position and quotas are upper bounds. In contrast to a pure house allocation problem, where an assignment is made on the basis of students' preferences over position types alone ${ }^{1}$ we assume that in a student placement problem additional information is available. ${ }^{2}$ For instance, college admissions of undergraduate students are often based on rankings obtained from one or several entrance exams. Then students who achieved higher test scores in the entrance exam of a certain college have higher priority for admission at that college than students with lower test scores. This situation can be described as a strict priority ranking of individuals for each position type. We call this collection of strict priority rankings a "priority structure." A placement mechanism "violates the priority of student $i$ for position $x$ " if there exists a preference profile under which student $i$ envies student $j$ who obtains $x$ even though $i$ has a higher priority for $x$ than $j$. A placement mechanism is fair if it never violates the specified priorities. Ergin (2002, Theorem 1) shows that a fair placement mechanism is efficient if and only if the priority structure is acyclic.

We also consider the student placement problem, but have in mind situations where students may not only care about their own position, but also about the position of their partners. The instance of couples may not be a prominent feature in undergraduate admissions, but it definitely is an issue for many couples who search for their first professional position in the same labor market, for instance medical students who look for residencies. We show that in the presence of couples, acyclicity is still a necessary condition for a placement mechanism to be fair and efficient, but it is not sufficient (Theorem 4.1). In order for a fair and efficient placement mechanism to exist, the priority structure also has to satisfy "priority-togetherness of couples" (Theorem 4.2). Loosely speaking, prioritytogetherness of a couple means that the members of the couple are ranked

\footnotetext{
1 Sometimes it is also assumed that exactly one position of each type is available. Some recent articles on house allocation problems are Ehlers (2002), Ehlers et al. (2002), and Ehlers and Klaus $(2003,2006 a, b)$.

2 See, for instance, Balinski and Sönmez (1999), Ergin (2002), and Kesten (2006).
} 
"close enough together" in the priority ranking of all position types. A priority structure is " $p t$-acyclic" if it satisfies acyclicity and priority-togetherness.

For student placement problems where all quotas are equal to one we characterize $p t$-acyclicity (Lemma 5.1) and show that it is a sufficient condition for the existence of a fair and efficient placement mechanism (Theorem 5.1). If in addition to $p t$-acyclicity we require reallocation- and vacancy-fairness for couples, the so-called dictator-bidictator placement mechanism is the unique fair and efficient placement mechanism (Theorem 5.2).

Finally, for general student placement problems, we show that $p t$-acyclicity may not be sufficient for the existence of a fair and efficient placement mechanism (Examples 5.4, 5.5, and 5.6). We identify a sufficient condition such that the so-called sequential placement mechanism produces a fair and efficient allocation (Theorem 5.3).

The paper is organized as follows. In Sect. 2, we introduce student placement problems with couples. In Sect. 3, we introduce efficiency, fairness, and acyclicity. In Sect. 4, we study necessary conditions for the compatibility of fairness and efficiency. Sect. 5 is devoted to sufficient conditions for the compatibility of fairness and efficiency. In Sect. 5.1 we focus on placement problems when all quotas are equal to one. In Sect. 5.2 we conclude with an extension of some of the results from Sect. 5.1 to general student placement problems. The proofs of all our results are relegated to the Appendix.

\section{Student placement with couples}

Let $N=\{1, \ldots, n\}$ denote the set of students. We assume that $N$ can be partitioned into a set of couples $C=\left\{c_{1}, \ldots, c_{k}\right\}$ such that for all $i \in\{1, \ldots, k\}$, $c_{i}=(2 i-1,2 i)$ and a set of single students $S=\{2 k+1, \ldots, n\}$. $^{3}$ Hence for a market with three couples and four single students, $N=\{1, \ldots, 10\}$ is composed of $C=\left\{c_{1}=(1,2), c_{2}=(3,4), c_{3}=(5,6)\right\}$ and $S=\{7,8,9,10\}$. If $C=\emptyset$, then our model coincides, for instance, with the model analyzed by Ergin (2002).

Let $X$ denote the finite set of position types that the students apply to. For any $x \in X$, let $q_{x} \geq 1$ denote the number of available positions, the quota, of position type $x$. Let $q \equiv\left(q_{x}\right)_{x \in X}$. Note that we simply refer to "position $x$ " when we mean one of the $q_{x}$ positions of position type $x$. Let 0 denote the null position, which does not belong to $X$ ("receiving the null position" means "not receiving any position"). Since the null position is freely available, we set $q_{0} \equiv \infty$.

Each single student $s \in S$ has an individual strict, transitive, and complete preference relation $R_{S}$ over $X \cup\{0\}$. Given $x, y \in X \cup\{0\}, x P_{S} y$ means that student $s$ strictly prefers $x$ to $y$. Let $\mathcal{R S}$ denote the set of all linear orders over $X \cup\{0\}$. In other words, $\mathcal{R S}$ equals the set of single students' preference

\footnotetext{
3 Note that all results can be straightforwardly extended to a model that includes triplets, quadruplets, etc.
} 
relations. Let $\mathcal{R} \mathcal{S}^{S}$ denote the set of all (preference) profiles $R_{S}=\left(R_{S}\right)_{s \in S}$ such that for all $s \in S, R_{s} \in \mathcal{R S}$.

Each couple $c \in C$ has an individual strict, transitive, and complete preference relation $R_{c}$ over all ordered pairs of feasible position type assignments $\mathcal{X} \equiv[(X \cup\{0\}) \times(X \cup\{0\})] \backslash\left\{(x, x): x \in X\right.$ and $\left.q_{x}=1\right\}$. To simplify notation, whenever we denote a position type assignment $(x, y)$ we always additionally assume that $(x, y)$ is feasible, i.e., $(x, y) \in \mathcal{X}$. This also means that all statements we make with respect to position type assignments only apply if the position type assignments in question are feasible. Given $c=(i, j),(x, y)$, and $\left(x^{\prime}, y^{\prime}\right)$, $(x, y) P_{c}\left(x^{\prime}, y^{\prime}\right)$ means that couple $c$ strictly prefers $(x, y)$, where $i$ is matched to $x$ and $j$ is matched to $y$, to $\left(x^{\prime}, y^{\prime}\right)$, where $i$ is matched to $x^{\prime}$ and $j$ is matched to $y^{\prime}$. Let $\mathcal{R C}$ denote the set of all linear orders over $\mathcal{X}$. In other words, $\mathcal{R C}$ equals the set of couples' preference relations. Let $\mathcal{R C}^{C}$ denote the set of all (preference) profiles $R_{C}=\left(R_{c}\right)_{c \in C}$ such that for all $c \in C, R_{c} \in \mathcal{R C}$.

We next introduce a subdomain of $\mathcal{R C}$ that allows us to relate a couple's preference relation to "individual preferences" of each member in a couple in a consistent way. Loosely speaking, this is the case when the unilateral improvement of one partner's position is considered beneficial for the couple as well. Couple $c=(i, j) \in C$ has responsive preferences if there exist preferences $R_{i}, R_{j} \in \mathcal{R} \mathcal{S}$ such that for all $x, y, z \in X \cup\{0\},\left[x P_{i} y\right.$ implies $\left.(x, z) P_{c}(y, z)\right]$ and [x $P_{j} y$ implies $\left.(z, x) P_{c}(z, y)\right] .{ }^{4}$ If these associated individual preferences $R_{i}$ and $R_{j}$ exist, then they are unique. Note that if a couple $c=(i, j)$ has responsive preferences, then one can easily derive the associated individual preferences $R_{i}$ and $R_{j}$. Let $\mathcal{R} \mathcal{R C} \subset \mathcal{R C}$ equal the set of couples' possible responsive preference relations. Let $\mathcal{R R C}^{C}$ denote the set of all responsive (preference) profiles $R_{C}=\left(R_{c}\right)_{c \in C}$ such that for all $c \in C, R_{c} \in \mathcal{R R C}$. For notational convenience we define $\mathcal{R} \equiv \mathcal{R C}^{C} \times \mathcal{R S}^{S}$ and $\mathcal{R} \mathcal{R} \equiv \mathcal{R} \mathcal{R} \mathcal{C}^{C} \times \mathcal{R S}^{S}$.

Let $x \in X$. We call a linear order $\succ_{x}$ over $N$ a priority ordering for position type $x$. A priority structure is a profile $\succ=\left(\succ_{x}\right)_{x \in X}$ specifying for each position type a priority ordering.

Since the set of students $N$, the set of position types $X$, their quotas $q$, and the priority structure $\succ$ are fixed, we denote a (student) placement problem by the students' preferences $R=\left(R_{C}, R_{S}\right) \in \mathcal{R}$. We assume that the null position is available in any placement problem.

When allocating positions each student either receives a "real position in $X$ " or the null position 0 . The null position can be assigned to several students without any restriction, but for all other positions the associated quotas are upper bounds. Formally, an allocation for $R$ is a list $\alpha=\left(\alpha_{i}\right)_{i \in N}$ such that for all $i \in N$, $\alpha_{i} \in X \cup\{0\}$, and for all $x \in X,\left|\left\{i \in N: \alpha_{i}=x\right\}\right| \leq q_{x}$. Thus, an allocation is by definition feasible. Note that not all available positions need to be assigned. Given $i \in N$, we call $\alpha_{i}$ the allotment of student $i$ at $\alpha$. Given $c=(i, j) \in C$, we call $\alpha_{c} \equiv\left(\alpha_{i}, \alpha_{j}\right)$ the allotment of couple $c$ at $\alpha$.

\footnotetext{
${ }^{4}$ Klaus and Klijn $(2005,2007)$ and Klaus et al. (2006) use the same notion of responsiveness in the context of two-sided matching. In Remark 5.2 we compare the role of responsiveness in two-sided matching and student placement.
} 
A (student) placement mechanism is a function $\varphi$ that assigns to each placement problem $R \in \mathcal{R}$ an allocation $\varphi(R)$.

\section{Efficiency, fairness, and acyclicity}

We are interested in student placement mechanisms that choose (Pareto) efficient allocations.

Definition 3.1 (Efficiency) A placement mechanism $\varphi$ is efficient if for all $R \in \mathcal{R}$, there is no allocation $\alpha=\left(\alpha_{i}\right)_{i \in N}$ for $R$ such that for all $p \in S \cup C, \alpha_{p} R_{p} \varphi_{p}(R)$ and for some $q \in S \cup C, \alpha_{q} P_{q} \varphi_{q}(R)$.

Next, we formulate the idea that an allocation may "violate the priority structure." First, we do so for single students. An allocation violates the priority of a single student $i \in S$ if there exists a position $x$ such that student $i$ has a higher priority for $x$ than one of the students assigned to it and student $i$ prefers to switch to position $x$.

Definition 3.2 (Fairness for single students; Balinski and Sönmez 1999) Given a placement problem $R \in \mathcal{R}, i \in S$, and a priority structure $\succ$, an allocation $\alpha$ for $R$ violates the priority of single student $i$ if there exist $x \in X$ and $k \in N \backslash\{i\}$ such that $\alpha_{k}=x, i \succ_{x} k$, and $x P_{i} \alpha_{i}$. A placement mechanism $\varphi$ is fair for single students if for all $R \in \mathcal{R}, \varphi(R)$ does not violate the priority of any single student.

Next, we list the three ways in which an allocation may violate the priority of a couple $c=(i, j) \in C$ :

(a) there exists a position $x \in X$ such that one member of the couple, e.g., student $i$, has a higher priority for $x$ than one of the students whose allotment is $x$ and who is not $i$ 's partner and couple $c$ prefers that $i$ switches to position $x$ while $j$ either keeps his/her allotment or switches to the null position.

(b) there exists a position $x \in X$ such that it is the allotment of one member of the couple, e.g., student $j$, the other member of the couple $i$ has a higher priority for $x$ than $j$, and couple $c$ prefers that $i$ switches to position $x$ and $j$ switches to the null position.

(c) there exist positions $x, y \in X$ (possibly of the same type) and two students $k, l \in N$ (possibly $k=j$ or $l=i$ ) such that $k$ 's allotment is $x, l$ 's allotment is $y, i$ has a higher priority for $x$ than $k, j$ has a higher priority for $y$ than $l$, and couple $c$ prefers that $i$ switches to $x$ and $j$ switches to $y^{5}$

\footnotetext{
5 The main idea of a fair allocation is that no single student (couple) can justifiably appeal for another position (assignment of positions) that would make him/her (it) better off. For single students only a position that is ranked higher than the allotment will make him/her better off. A couple has various possibilities to improve its allotment: one or both partners could change their positions, one partner could change his/her position while the other partner switches to the null position at the same time, or one partner changes his/her position while the other partner receives his/her previous position. Here we only consider violations of a couple's priorities that would lead to a
} 
Definition 3.3 (Fairness for couples) Given a placement problem $R \in \mathcal{R},(i, j) \in C$, and a priority structure $\succ$, an allocation $\alpha$ for $R$ violates the priority of couple $(i, j)$ if

(a.1) there exist $x \in X$ and $k \in N \backslash\{i, j\}$ such that $\alpha_{k}=x, i \succ_{x} k$, and $\left[\left(x, \alpha_{j}\right) P_{(i, j)}\left(\alpha_{i}, \alpha_{j}\right)\right.$ or $\left.(x, 0) P_{(i, j)}\left(\alpha_{i}, \alpha_{j}\right)\right]$, or

(a.2) there exist $x \in X$ and $k \in N \backslash\{i, j\}$ such that $\alpha_{k}=x, j \succ_{x} k$, and $\left[\left(\alpha_{i}, x\right) P_{(i, j)}\left(\alpha_{i}, \alpha_{j}\right)\right.$ or $\left.(0, x) P_{(i, j)}\left(\alpha_{i}, \alpha_{j}\right)\right]$, or

(b.1) there exists $x \in X$ such that $\alpha_{j}=x, i \succ_{x} j$, and $(x, 0) P_{(i, j)}\left(\alpha_{i}, x\right)$, or

(b.2) there exists $x \in X$ such that $\alpha_{i}=x, j \succ_{x} i$, and $(0, x) P_{(i, j)}\left(x, \alpha_{j}\right)$, or

(c) there exist $x, y \in X$ and $k, l \in N, k \neq l$, such that $\alpha_{k}=x, \alpha_{l}=y, i \succ_{x} k$, $j \succ_{y} l$, and $(x, y) P_{(i, j)}\left(\alpha_{i}, \alpha_{j}\right)$.

A placement mechanism $\varphi$ is fair for couples if for all $R \in \mathcal{R}, \varphi(R)$ does not violate the priority of any couple.

Definition 3.4 (Fairness) A placement mechanism is fair if it is fair for single students and couples.

We next introduce an acyclicity condition, due to Ergin (2002), that turns out to be crucial for the existence of a fair and efficient placement mechanism.

Definition 3.5 (Cycles and acyclicity) Given a priority structure $\succ$, a cycle is constituted of distinct $x, y \in X$ and $i, j, k \in N$ such that the following two conditions are satisfied:

cycle condition: $i \succ_{x} j \succ_{x} k$ and $k \succ_{y} i$ and

$c$-scarcity condition: there exist disjoint sets $N_{x}, N_{y} \subseteq N \backslash\{i, j, k\}$ (possibly $N_{x}=\emptyset$ or $\left.N_{y}=\emptyset\right)$ such that $N_{x} \subseteq\left\{l \in N: l \succ_{x} j\right\}, N_{y} \subseteq\left\{l \in N: l \succ_{y} i\right\}$, $\left|N_{x}\right|=q_{x}-1$, and $\left|N_{y}\right|=q_{y}-1$.

A priority structure is acyclic if no cycles exist.

If quotas are all equal to 1 , then the cycle condition is sufficient to establish the existence of a cycle. For other quotas, the $c$-scarcity condition limits the definition of a cycle to cases where there indeed exist placement problems in $\mathcal{R}$ such that students $i, j$, and $k$ compete for position types $x$ and $y$ (in the absence of this competition, e.g., because the quotas do in fact not limit the access of the students to positions of type $x$ and $y$, a cycle will not lead to the violation of efficiency or the given priorities - see Ergin 2002, for further discussion).

In the sequel we will sometimes write "D $x$ " instead of "Definition $x$ ".

better allotment for the couple because one or both partners receive positions for which they have a higher priority and possibly one partner chooses the null position. Since students do not have any property rights over positions, passing on a position to a partner or adopting a vacant position may not be allowed for a couple and therefore violations that give better allotments conditioned on such transactions are not considered in our basic fairness concept, but later in two extra fairness conditions for couples (Definitions 5.4 and 5.5). 


\section{Fairness and efficiency for student placement with couples: necessary conditions}

In the absence of couples, Ergin (2002) shows that the acyclicity of the priority structure is a necessary and sufficient condition for a placement mechanism to be fair and efficient. He shows that under the assumption of an acyclic priority structure, the unique efficient placement mechanism that is fair can be found using a simple adaptation of the so-called deferred acceptance algorithm (Gale and Shapley 1962). Theorem 1 in Ergin (2002) implies the following result.

Corollary 4.1 (Ergin 2002) Let $C=\emptyset$.

A fair and efficient placement mechanism $\varphi$ exists (and is unique) if and only if $\succ$ is acyclic.

Our first result is that for placement problems with couples, acyclicity is still a necessary, but not a sufficient condition for the existence of a fair and efficient placement mechanism.

Theorem 4.1 (Necessity of acyclicity)

(a) A placement mechanism $\varphi$ is fair and efficient only if $\succ$ is acyclic.

(b) The acyclicity of $\succ$ is not a sufficient condition for the existence of a fair and efficient placement mechanism $\varphi$.

In the proof of Theorem 4.1 (Appendix) we use only responsive preferences for couples. Hence, Theorem 4.1 remains valid if we restrict couples' preferences to be responsive.

Remark 4.1 In order to prove Theorem 4.1(a) we assume that acyclicity is violated and derive a contradiction to fairness and efficiency. For any violation of acyclicity that only involves single students, the proof of Theorem 4.1(a) essentially equals the proof of Corollary 4.1 (Ergin 2002,Theorem 1). However, extending the proof to situations where couples are involved in a violation of acyclicity requires substantial extra work. The key then is to define adequate individual preferences not only for single students, but also for the members of all couples and, in addition, corresponding couples' preferences.

Next, in order for fairness and efficiency to be compatible, we need an additional condition on the priority structure: members of a couple have to be "close enough" in the priority structure. In the following definition we formalize what we mean by "close enough."

Definition 4.1 (Priority-separation, priority-togetherness, and pt-acyclicity) Let $c=(i, j) \in C$. Given a priority structure $\succ$, couple $c$ is priority-separated if there exists $x \in X$ and $k \in N$ such that the following two conditions are satisfied: weak priority-separation

(i) if $i \succ_{x} j$, then $i \succ_{x} k \succ_{x} j$, and

(ii) if $j \succ_{x}$ i, then $j \succ_{x} k \succ_{x} i$, and 
$p s$-scarcity condition

there exists a set $\bar{N}_{x} \subseteq N \backslash\{i, j, k\}$ (possibly $\bar{N}_{x}=\emptyset$ ) such that

(i) if $i \succ_{x} j$, then $\bar{N}_{x} \subseteq\left\{l \in N: l \succ_{x} j\right\}$ and $\left|\bar{N}_{x}\right|=q_{x}-1$, and

(ii) if $j \succ_{x}$, then $\bar{N}_{x} \subseteq\left\{l \in N: l \succ_{x} i\right\}$ and $\left|\bar{N}_{x}\right|=q_{x}-1$.

A priority structure satisfies priority-togetherness (of couples) if no couple is priority-separated. A priority structure is pt-acyclic if it is acyclic and satisfies priority-togetherness.

If quotas are all equal to 1 , then weak priority-separation is sufficient to define priority-separation of couples. For other quotas, the $p s$-scarcity condition limits the definition of priority-separation to cases where there indeed exist placement problems in $\mathcal{R}$ such that couple $c$ and student $k$ compete for position type $x$ (in the absence of this competition, e.g., because the quotas do in fact not limit the access of the students to position type $x$, a priority-separation will not lead to the violation of efficiency or the given priorities).

Theorem 4.2 (Necessity of $p t$-Acyclicity) A placement mechanism $\varphi$ is fair and efficient only if $\succ$ is pt-acyclic.

Note that in the proof of Theorem 4.2 (Appendix) we use only responsive preferences for couples. Hence, Theorem 4.2 remains valid if we restrict couples' preferences to be responsive.

\section{Fairness and efficiency for student placement with couples: sufficiency and uniqueness}

Our next goal is to introduce a fair and efficient placement mechanism for $p t$-acyclic priority structures. First we show that any priority structure defines in a natural way a partition of the students. As will turn out, the structure of the partition induced by a $p t$-acyclic priority structure makes it possible to define fair and efficient placement mechanisms.

Given position type $x \in X$ and a subset $N^{\prime} \subseteq N$ of students, let top $\left(\succ_{x}, N^{\prime}\right)$ denote the student with the highest priority for position type $x$ among the students in $N^{\prime}$, i.e., $i=\operatorname{top}\left(\succ_{x}, N^{\prime}\right)$ if and only if $i \in N^{\prime}$ and for all $j \in N^{\prime} \backslash\{i\}$, $i \succ_{x} j$. Given a subset $N^{\prime} \subseteq N$ of students, let top $\left(N^{\prime}\right)$ denote the set of students with the highest priority for some position type among the students in $N^{\prime}$, i.e., $\operatorname{top}\left(N^{\prime}\right) \equiv \bigcup_{x \in X} \operatorname{top}\left(\succ_{x}, N^{\prime}\right)$. Using this notation, we define a partition of the set of all students $N$ that is induced by a (not necessarily $p t$-acyclic) priority structure $\succ$ :

$S_{1}$ denotes the set of students who have the highest priority for some position type; $S_{2}$ denotes the set of students in the remaining set of students $N \backslash S_{1}$ who now have the highest priority for some position type, etc. Given $S_{k}$, we interpret the index $k$ as a level of priority: students in $S_{1}$ have the highest possible priority, students in $S_{2}$ have the next highest priority, etc. 
Definition 5.1 (Partition $\mathcal{S}$ induced by $\succ$ ) Let $S_{1} \equiv \operatorname{top}(N)$ and for $k>1$, if $N \backslash \bigcup_{l=1}^{k-1} S_{l} \neq \emptyset$, then $S_{k} \equiv \operatorname{top}\left(N \backslash \bigcup_{l=1}^{k-1} S_{l}\right) \neq \emptyset$. Hence, there exists $p \geq 1$, $p<\infty$, such that for all $k \in\{1, \ldots, p\}, S_{k} \neq \emptyset$, and $N \backslash\left[\bigcup_{l=1}^{p} S_{l}\right]=\emptyset$. By $\mathcal{S} \equiv\left\{S_{1}, \ldots, S_{p}\right\}$, we define the partition (of $N$ ) induced by $\succ$, i.e., $N=\bigcup_{l=1}^{p} S_{l}$ and for $k, k^{\prime} \in\{1, \ldots, p\}, k \neq k^{\prime}, S_{k} \cap S_{k^{\prime}}=\emptyset$.

\subsection{Sufficiency and uniqueness when all quotas are equal to one}

Throughout this subsection we assume that for all $x \in X, q_{x}=1$ and refer to position $x$ instead of position type $x$. For this special situation, $p t$-acyclicity is equivalent to the following.

Definition 5.2 ( $p t$-acyclicity when all quotas are equal to one) A priority structure $\succ$ is pt-acyclic if it has neither cycles nor weakly priority-separated couples:

no cycles: there exist no $i, j, k \in N$ and $x, y \in X$ such that $i \succ_{x} j \succ_{x} k$ and $k \succ_{y} i$ and

no weak priority-separation: there exist no $(i, j) \in C, k \in N$, and $x \in X$ such that $i \succ_{x} k \succ_{x} j$ or $j \succ_{x} k \succ_{x} i$.

The next lemma describes the implications that $p t$-acyclicity of a priority structure has on its induced partition. First, (ia) each component of the partition either contains one or two students, that is, at most two students share the same level of priority. Second, (ib) if two students $i, j$ share the same level of priority, then they are neighbors in the priority structure, i.e., for all positions, either $i$ is ranked just after $j$ or $j$ is ranked just after $i$. Finally, members of a couple $c=(i, j)$ either (iia) share the same level of priority or (iib and iic) they do not share the level of priority with any other student(s), but they have consecutive levels of priority.

Lemma 5.1 Priority structure $\succ$ is pt-acyclic if and only if partition $\mathcal{S}=$ $\left\{S_{1}, \ldots, S_{p}\right\}$ has the following properties:

(i) for all $k \in\{1, \ldots, p\}$,

(a) $\left|S_{k}\right| \leq 2$

(b) if $S_{k}=\{i, j\}, i \neq j$, then for any $x \in X$,

$$
\left[i=\operatorname{top}\left(x, N \backslash \bigcup_{l=1}^{k-1} S_{l}\right) \text { implies } j=\operatorname{top}\left(x, N \backslash\left[\bigcup_{l=1}^{k-1} S_{l} \cup\{i\}\right]\right)\right] \text { and }
$$

(ii) for all $c=(i, j) \in C$, if for $k \in\{1, \ldots, p\}, S_{k} \cap\{i, j\} \neq \emptyset$, then

(a) $S_{k}=\{i, j\}$, or

(b) $S_{k} \cup S_{k-1}=\{i, j\}$, or

(c) $S_{k} \cup S_{k+1}=\{i, j\}$.

In the proof of Lemma 5.1 (Appendix) we show that condition (i) characterizes the acyclicity of priority structure $\succ$, and that given acyclicity, condition (ii) characterizes priority-togetherness. The following direct implications of condition (i) of Lemma 5.1 turn out to be useful later on. 
Lemma 5.2 Let $\mathcal{S}$ be the partition induced by $\succ$ and assume $\mathcal{S}$ satisfies (i) in Lemma 5.1.

(a) Let $x \in X$ and $i, \vec{i}, i^{\prime} \in N$ be such that $i \succ_{x} \vec{i} \succ_{x} i^{\prime}$. Furthermore, let $k, k^{\prime} \in$ $\{1, \ldots, p\}$ be such that $i \in S_{k}$ and $i^{\prime} \in S_{k^{\prime}}$. Then, $k<k^{\prime}$.

(b) If $i \in S_{k}, i^{\prime} \in S_{k^{\prime}}, k, k^{\prime} \in\{1, \ldots, p\}$, and $k<k^{\prime}$, then for all $x \in X, i \succ_{x} i^{\prime}$.

We can now use partition $\mathcal{S}$ to define the dictator-bidictator deferred acceptance placement mechanism, the DB placement mechanism for short. Informally, the DB placement mechanism works as follows. Determine who has the highest level of priority. If a single student has the highest priority, then he/she receives his/her most preferred position. If two single students share the highest priority, then either they receive their most preferred positions or, in case of a conflict, the priority decides who gets his/her most preferred position and who gets his/her second most preferred position. If a member of a couple has the highest level of priority or a couple shares the highest level of priority, then the couple receives its most preferred position assignment. Repeat this step with the remaining students and positions, i.e., determine who has the next highest level of priority, etc.

Let $Y \subseteq X$ be a set of positions. Let $s \in S$ and $R_{s} \in \mathcal{R S}$. Then $\operatorname{top}\left(R_{S}, Y\right)$ denotes student $s$ 's most preferred position in $Y \cup\{0\}$, i.e., $y=\operatorname{top}\left(R_{S}, Y\right) \in$ $Y \cup\{0\}$ if and only if for all $x \in Y \cup\{0\}, x \neq y, y P_{s} x$. Let $c \in C$ and $R_{c} \in$ $\mathcal{R C}$. Then $\operatorname{top}\left(R_{c}, Y\right)$ denotes couple $c$ 's most preferred position assignment in $\mathcal{Y} \equiv[(Y \cup\{0\}) \times(Y \cup\{0\})] \backslash\{(y, y): y \in Y\}$, i.e., $\left(y, y^{\prime}\right)=\operatorname{top}\left(R_{c}, Y\right) \in \mathcal{Y}$ if and only if for all $\left(x, x^{\prime}\right) \in \mathcal{Y},\left(x, x^{\prime}\right) \neq\left(y, y^{\prime}\right),\left(y, y^{\prime}\right) P_{c}\left(x, x^{\prime}\right)$.

Definition 5.3 (The dictator-bidictator (DB) placement mechanism $\varphi^{\mathcal{S}}$ ) Let $\succ$ be a pt-acyclic priority structure that induces partition $\mathcal{S}=\left\{S_{1}, \ldots, S_{p}\right\}$. Given $R \in \mathcal{R}$, we calculate $\varphi^{\mathcal{S}}(R)$ as follows. Set $k=1$ and $X_{1} \equiv X$. As long as $k \leq p$, do Step $k$ :

(a) $S_{k}=\{s\}$ and $s \in S: \varphi_{s}^{\mathcal{S}}(R) \equiv \operatorname{top}\left(R_{s}, X_{k}\right), X_{k+1} \equiv X_{k} \backslash\left\{\varphi_{s}^{\mathcal{S}}(R)\right\}$, and set $k=k+1$.

If a single student has the highest priority for all remaining real positions in $X_{k}$, he/she chooses his/her favorite position in $X_{k} \cup\{0\}$. The set of unassigned real positions equals $X_{k+1}$.

(b) $S_{k}=\{s\}, s \notin S$, and $S_{k} \cup S_{k+1}=\left\{s, s^{\prime}\right\}$ such that $c=\left(s, s^{\prime}\right) \in C$ or $c=\left(s^{\prime}, s\right) \in$ $C: \varphi_{c}^{\mathcal{S}}(R) \equiv \operatorname{top}\left(R_{c}, X_{k}\right), X_{k+2} \equiv X_{k} \backslash\left\{\varphi_{s}^{\mathcal{S}}(R), \varphi_{s^{\prime}}^{\mathcal{S}}(R)\right\}$, and set $k=k+2$.

If the member of a couple has the highest priority for all remaining real positions in $X_{k}$, then the couple chooses its most favorite position assignment in $\mathcal{X}_{k}$. The set of unassigned real positions equals $X_{k+2}$.

(c) $S_{k}=\left\{s, s^{\prime}\right\}$ and $s, s^{\prime} \in S$ :

(c.1) if top $\left(R_{s}, X_{k}\right) \neq \operatorname{top}\left(R_{s^{\prime}}, X_{k}\right)$ or $\operatorname{top}\left(R_{s}, X_{k}\right)=\operatorname{top}\left(R_{S^{\prime}}, X_{k}\right)=0, \varphi_{s}^{\mathcal{S}}(R) \equiv$ $\operatorname{top}\left(R_{s}, X_{k}\right), \varphi_{s^{\prime}}^{\mathcal{S}}(R) \equiv \operatorname{top}\left(R_{S^{\prime}}, X_{k}\right), X_{k+1} \equiv X_{k} \backslash\left\{\varphi_{s}^{\mathcal{S}}(R), \varphi_{s^{\prime}}^{\mathcal{S}}(R)\right\}$, and set $k=$ $k+1$.

(c.2) if $\operatorname{top}\left(R_{s}, X_{k}\right)=\operatorname{top}\left(R_{s^{\prime}}, X_{k}\right) \equiv \hat{x} \in X$ and $s \succ_{\hat{x}} s^{\prime}, \varphi_{s}^{\mathcal{S}}(R) \equiv \operatorname{top}\left(R_{s}, X_{k}\right)$, $\varphi_{\mathcal{S}^{\prime}}^{\mathcal{S}}(R) \equiv \operatorname{top}\left(R_{\mathcal{S}^{\prime}}, X_{k} \backslash\left\{\varphi_{s}^{\mathcal{S}}(R)\right\}\right), X_{k+1} \equiv X_{k} \backslash\left\{\varphi_{s}^{\mathcal{S}}(R), \varphi_{S^{\prime}}^{\mathcal{S}}(R)\right\}$, and set $k=k+1$. 
(c.3) if $\operatorname{top}\left(R_{s}, X_{k}\right)=\operatorname{top}\left(R_{s^{\prime}}, X_{k}\right) \equiv \hat{x} \in X$ and $s^{\prime} \succ_{\hat{x}} s, \varphi_{s^{\prime}}^{\mathcal{S}}(R) \equiv \operatorname{top}\left(R_{s^{\prime}}, X_{k}\right)$, $\varphi_{s}^{\mathcal{S}}(R) \equiv \operatorname{top}\left(R_{s}, X_{k} \backslash\left\{\varphi_{s^{\prime}}^{\mathcal{S}}(R)\right\}\right), X_{k+1} \equiv X_{k} \backslash\left\{\varphi_{s}^{\mathcal{S}}(R), \varphi_{s^{\prime}}^{\mathcal{S}}(R)\right\}$, and set $k=k+1$.

If two single students share the highest priorities for all remaining real positions in $X_{k}$, then there are two possibilities. First, if feasible, both of them choose their favorite position in $X_{k} \cup\{0\}$; see (c.1). Second, if both of them prefer the same real position in $X_{k}$, then the student with the higher priority receives it and the remaining student is assigned his/her second best position in $X_{k} \cup\{0\}$; see (c.2) and (c.3). The set of unassigned real positions equals $X_{k+1}$.

(d) $S_{k}=\left\{s, s^{\prime}\right\}$ and $c=\left(s, s^{\prime}\right) \in C$ or $c=\left(s^{\prime}, s\right) \in C: \varphi_{c}^{\mathcal{S}}(R) \equiv \operatorname{top}\left(R_{c}, X_{k}\right)$, $X_{k+1} \equiv X_{k} \backslash\left\{\varphi_{s}^{\mathcal{S}}(R), \varphi_{s^{\prime}}^{\mathcal{S}}(R)\right\}$, and set $k=k+1$.

If the two members of a couple together have the highest priority for all remaining real positions in $X_{k}$, then the couple chooses its most favorite position assignment in $\mathcal{X}_{k}$. The set of unassigned real positions equals $X_{k+1}$.

The allocation $\varphi^{\mathcal{S}}(R)$ is obtained after at most p steps.

Example 5.1 (An application of the DB placement mechanism) Let $N=$ $\{1, \ldots, 8\}$ be such that $C=\left\{c_{1}=(1,2), c_{2}=(3,4)\right\}$ and $S=\{5,6,7,8\}$; and $X=\left\{x_{1}, \ldots, x_{7}\right\}$ with quota 1 for each position type. We depict the $p t$-acyclic priority structure $\succ$ and preference profile $R \in \mathcal{R}$ in the two tables below.

\begin{tabular}{lllllll}
\hline$\succ_{x_{1}}$ & $\succ_{x_{2}}$ & $\succ_{x_{3}}$ & $\succ_{x_{4}}$ & $\succ_{x_{5}}$ & $\succ_{x_{6}}$ & $\succ_{x_{7}}$ \\
\hline 6 & 6 & 6 & 6 & 6 & 6 & 6 \\
2 & 1 & 1 & 2 & 1 & 2 & 2 \\
1 & 2 & 2 & 1 & 2 & 1 & 1 \\
7 & 5 & 7 & 5 & 5 & 5 & 7 \\
5 & 7 & 5 & 7 & 7 & 7 & 5 \\
4 & 4 & 4 & 4 & 4 & 4 & 4 \\
3 & 3 & 3 & 3 & 3 & 3 & 3 \\
8 & 8 & 8 & 8 & 8 & 8 & 8 \\
\hline
\end{tabular}

\begin{tabular}{llllll}
\hline$R_{c_{1}}$ & $R_{c_{2}}$ & $R_{5}$ & $R_{6}$ & $R_{7}$ & $R_{8}$ \\
\hline$\left(x_{3}, x_{1}\right)$ & $\left(x_{2}, x_{1}\right)$ & $x_{1}$ & $x_{3}$ & $x_{1}$ & $x_{1}$ \\
$\left(x_{1}, x_{3}\right)$ & $\left(x_{4}, x_{5}\right)$ & $x_{2}$ & $x_{1}$ & $x_{2}$ & $x_{2}$ \\
$\left(x_{2}, x_{1}\right)$ & $\left(x_{6}, x_{5}\right)$ & $x_{3}$ & $x_{2}$ & $x_{3}$ & $x_{3}$ \\
$\left(x_{1}, x_{2}\right)$ & $\left(x_{7}, x_{5}\right)$ & $x_{4}$ & $x_{4}$ & $x_{4}$ & $x_{4}$ \\
$\left(x_{3}, x_{2}\right)$ & $\left(0, x_{5}\right)$ & $x_{5}$ & $x_{7}$ & $x_{5}$ & $x_{5}$ \\
$\left(x_{2}, x_{3}\right)$ & $\left(x_{5}, x_{7}\right)$ & $x_{6}$ & 0 & $x_{6}$ & $x_{6}$ \\
$\left(x_{3}, 0\right)$ & $\left(x_{4}, x_{7}\right)$ & $x_{7}$ & $x_{5}$ & $x_{7}$ & $x_{7}$ \\
$\left(0, x_{3}\right)$ & $\left(x_{6}, x_{7}\right)$ & 0 & $x_{6}$ & 0 & 0 \\
$\left(x_{1}, 0\right)$ & $\left(0, x_{7}\right)$ & & & & \\
$\left(0, x_{1}\right)$ & $(0,0)$ & & & & \\
$\cdots$ & $\ldots$ & & & &
\end{tabular}

In the table denoting priority structure $\succ$, students with higher priority for a position are denoted above students with lower priorities, e.g., $6 \succ_{x_{1}} 2 \succ_{x_{1}} 1 \succ_{x_{1}}$ $7 \succ_{x_{1}} 5 \succ_{x_{1}} 4 \succ_{x_{1}} 3 \succ_{x_{1}}$. In the table denoting preference profile $R$, position assignments that are more preferred are denoted above less preferred position 
assignments, e.g., $\left(x_{3}, x_{1}\right) R_{c_{1}}\left(x_{1}, x_{3}\right) R_{c_{1}}\left(x_{2}, x_{1}\right) R_{c_{1}}\left(x_{1}, x_{2}\right) R_{c_{1}}\left(x_{3}, x_{2}\right) R_{c_{1}} \ldots$ or $x_{1} R_{5} x_{2} R_{5} x_{3} R_{5} x_{4} R_{5} x_{5} R_{5} \ldots$

Priority structure $\succ$ induces partition $S=\left\{S_{1}, S_{2}, S_{3}, S_{4}, S_{5}, S_{6}\right\}$ with $S_{1}=\{6\}$, $S_{2}=\{1,2\}, S_{3}=\{5,7\}, S_{4}=\{4\}, S_{5}=\{3\}$, and $S_{6}=\{8\}$. Note that $S_{4} \cup S_{5}=\{3,4\}$ consists of couple $c_{2}$. We now calculate $\varphi^{\mathcal{S}}(R)$. Recall that $X_{1}=X$.

Step 1: $S_{1}=\{6\}$ and $6 \in S: \varphi_{6}^{\mathcal{S}}(R)=x_{3}$ and $X_{2}=\left\{x_{1}, x_{2}, x_{4}, x_{5}, x_{6}, x_{7}\right\}$.

Step 2: $S_{2}=\{1,2\}$ and $(1,2) \in C: \varphi_{c_{1}}(R)=\left(x_{2}, x_{1}\right)$ and $X_{3}=\left\{x_{4}, x_{5}, x_{6}, x_{7}\right\}$.

Step 3: $S_{3}=\{5,7\}$ and 5,7 $\in S: \varphi_{5}^{\mathcal{S}}(R)=x_{4}, \varphi_{7}^{\mathcal{S}}(R)=x_{5}$, and $X_{4}=\left\{x_{6}, x_{7}\right\}$.

Step 4: $S_{4}=\{4\}, S_{5}=\{3\}$, and $(3,4) \in C: \varphi_{c_{2}}^{\mathcal{S}}(R)=\left(x_{6}, x_{7}\right)$ and $X_{6}=\emptyset$.

Step 6: $S_{6}=\{8\}$ and $8 \in S: \varphi_{8}^{\mathcal{S}}(R)=0$ and $X_{7}=\emptyset$.

Hence, $\varphi^{\mathcal{S}}(R)=\left(x_{2}, x_{1}, x_{6}, x_{7}, x_{4}, x_{3}, x_{5}, 0\right)$.

Our next result is that for $p t$-acyclic priority structures the DB placement mechanism is fair and efficient.

Theorem 5.1 (Quotas equal to one: sufficiency of pt-acyclicity) Let $\succ$ be a pt-acyclic priority structure. Then, the $D B$ placement mechanism $\varphi^{\mathcal{S}}$ is fair and efficient.

Remark 5.1 Theorem 5.1 is implied by one of our later results for general placement problems (Theorem 5.3). In the proof of Theorem 5.3 we use a result from Ergin (2002). However, a self-contained proof of Theorem 5.1 is available from any of the authors upon request.

The following two examples show exactly why $p t$-acyclicity is not strong enough to obtain the DB Placement Mechanism as the unique fair and efficient mechanism.

Example 5.2 (Fairness, efficiency, and reallocation) Let $N=\{1,2,3\}$ be such that $C=\{c=(1,2)\}$ and $S=\{3\}$; and $X=\{x, y, z\}$ with quota 1 for each position type. Let $\succ$ be such that for all $x^{\prime} \in X, 1 \succ_{x^{\prime}} 2 \succ_{x^{\prime}}$ 3. Consider $R_{c} \in \mathcal{R} \mathcal{R C}$ and $R_{3} \in \mathcal{R S}$ such that $(x, y) P_{c}(y, z) P_{c} \ldots$ and $x P_{3}$ y $P_{3} z P_{3} 0$, where the "tail" of $R_{c}$, denoted by "...", can be any fixed linear order of the remaining position assignments such that couple $c$ 's preferences are responsive. Let $R=\left(R_{c}, R_{3}\right)$. It is easy to check that $S_{i}=\{i\}$ and hence $\varphi^{\mathcal{S}}(R)=(x, y, z)$. However, allocation $\alpha=(y, z, x)$ is also fair and efficient.

Suppose that we allow couples to reallocate positions among themselves. Then, we could argue that allocation $\alpha$ "violates the priority of couple $c$ after reallocation" since student 1 can pass on position $y$ to his/her partner and then complain that his/her priority for position $x$ is violated because $1 \succ_{x} 3$. Note that by passing position $y$ to student 2 , student 3 's priority is not violated because $2 \succ_{y} 3$ (since student 2 is student 1 's partner, $1 \succ_{y} 2$ is not relevant).

Example 5.3 (Fairness, efficiency, and vacancies) Let $N=\{1,2,3\}$ be such that $C=\{c=(1,2)\}$ and $S=\{3\}$; and $X=\{x, y, z, q\}$ with quota 1 for each position type. Let $\succ$ be such that for all $x^{\prime} \in X, 1 \succ_{x^{\prime}} 2 \succ_{x^{\prime}} \quad 3$. Consider $R_{c} \in \mathcal{R C}$ and $R_{3} \in \mathcal{R S}$ such that $(x, y) P_{c}(z, q) P_{c} \ldots$ and $x P_{3}$ y $P_{3} z P_{3} q P_{3} 0$, where the "tail" 
of $R_{c}$, denoted by “...", can be any fixed linear order of the remaining position assignments. Let $R=\left(R_{c}, R_{3}\right)$. It is easy to check that $S_{i}=\{i\}$ and hence $\varphi^{\mathcal{S}}(R)=(x, y, z)$. However, allocation $\alpha=(z, q, x)$ is also fair and efficient.

Suppose that we allow students to occupy a vacant position. Then, we could argue that allocation $\alpha$ "violates the priority of couple $c$ when taking into account vacancies" since student 2 can claim position $y$ and then his/her partner can complain that his/her priority for position $x$ is violated because $1 \succ_{x} 3$. Note that student 2's occupation of position $y$ does not violate student 3's priority.

Definition 5.4 (Reallocation-fairness for couples) Given a placement problem $R \in \mathcal{R}, i \in N$, and a priority structure $\succ$, an allocation $\alpha$ for $R$ violates the priority of couple $(i, j) \in C$ after reallocation if

(r.1) $\alpha_{i} \in X$ and there exist $x \in X$ and $k \in N \backslash\{i, j\}$ such that $\alpha_{k}=x, i \succ_{x} k$, and $\left(x, \alpha_{i}\right) P_{(i, j)}\left(\alpha_{i}, \alpha_{j}\right)$ or

(r.2) $\alpha_{j} \in X$ and there exist $x \in X$ and $k \in N \backslash\{i, j\}$ such that $\alpha_{k}=x, j \succ_{x} k$, and $\left(\alpha_{j}, x\right) P_{(i, j)}\left(\alpha_{i}, \alpha_{j}\right)$.

A placement mechanism $\varphi$ is reallocation-fair (for couples) if for all $R \in \mathcal{R}$, $\varphi(R)$ does not violate the priority of any couple after reallocation.

Definition 5.5 (Vacancy-fairness for couples) Given a placement problem $R \in \mathcal{R}, i \in N$, and a priority structure $\succ$, an allocation $\alpha$ for $R$ violates the priority of couple $(i, j) \in C$ when taking vacancies into account if

(v.1) there exist $x \in X, k \in N \backslash\{i, j\}$, and $v \in X \backslash\left\{\alpha_{l}: l \in N\right\}$ such that $\alpha_{k}=x$, $i \succ_{x} k$, and $(x, v) P_{(i, j)}\left(\alpha_{i}, \alpha_{j}\right)$ or

(v.2) there exist $x \in X, k \in N \backslash\{i, j\}$, and $v \in X \backslash\left\{\alpha_{l}: l \in N\right\}$ such that $\alpha_{k}=x$, $j \succ_{x} k$, and $(v, x) P_{(i, j)}\left(\alpha_{i}, \alpha_{j}\right)$.

A placement mechanism $\varphi$ is vacancy-fair (for couples) if for all $R \in \mathcal{R}, \varphi(R)$ does not violate the priority of any couple when taking vacancies into account.

It is easy to check that the DB placement mechanism is reallocation- and vacancy-fair (for $p t$-acyclic priority structures). Given a $p t$-acyclic priority structure a reallocation- and vacancy-fair placement mechanism is fair and efficient if and only if it is the DB placement mechanism.

Theorem 5.2 (Quotas equal to one: uniqueness of the DB placement mechanism) Let $\succ$ be a pt-acyclic priority structure. Let $\varphi$ be a reallocation- and vacancy-fair placement mechanism. Then, $\varphi$ is fair and efficient if and only if $\varphi=\varphi^{\mathcal{S}}$.

Note that the proof (Appendix) of Theorem 5.2 remains the same for the domain of responsive preferences. Hence, Theorem 5.2 remains valid if we restrict couples' preferences to be responsive.

In Example 5.2 we exhibit a placement problem with responsive preferences such that there is an allocation that violates the priority of a couple after reallocation. In Example 5.3 we cannot exhibit a placement problem with responsive 
preferences such that there is an allocation that violates the priority of a couple when taking vacancies into account. The following result explains why.

Proposition 5.1 Let $\varphi$ be a placement mechanism on the domain of responsive preferences $\mathcal{R} \mathcal{R}$. If $\varphi$ is fair and efficient (on $\mathcal{R} \mathcal{R}$ ), then $\varphi$ is also vacancy-fair (on $\mathcal{R} \mathcal{R})$.

Corollary 5.1 Let $\succ$ be a pt-acyclic priority structure. Let $\varphi$ be a reallocation-fair placement mechanism on the domain of responsive preferences $\mathcal{R} \mathcal{R}$. Then, $\varphi$ is fair and efficient (on $\mathcal{R} \mathcal{R})$ if and only if $\varphi=\varphi^{\mathcal{S}}($ on $\mathcal{R} \mathcal{R})$.

\subsection{Sufficiency: the general case}

First, we demonstrate with three examples that if quotas are not all equal to one, then $p t$-acyclicity may not be sufficient for the existence of a fair and efficient placement mechanism. All three examples are minimal in the sense that the number of students and couples are as small as possible.

Example 5.4 ( $p t$-acyclic priorities and no fair and efficient allocation I) Let $N=$ $\{1,2,3\}$ be such that $C=\left\{c_{1}=(1,2)\right\}$ and $S=\{3\}$; and $X=\{x, y\}$ with $q_{x}=1$ and $q_{y}=2$. Let $\succ$ be such that $1 \succ_{x} 2 \succ_{x} 3$ and $1 \succ_{y} 3 \succ_{y} 2$. Note that $\succ$ is $p t$-acyclic. Consider $R_{c_{1}} \in \mathcal{R C}$ and $R_{3} \in \mathcal{R S}$ such that $(y, y) P_{c_{1}}(x, y) P_{c_{1}}(0,0) P_{c_{1}}$ ... and y $P_{3} 0 P_{3} x$, where the "tail" of $R_{c_{1}}$, denoted by "...", can be any fixed linear order of the remaining position type assignments. The only efficient allocations for placement problem $R=\left(R_{c_{1}}, R_{3}\right)$ are $\alpha=((y, y), 0)$ and $\beta=((x, y), y)$. Since $3 \succ_{y} 2$ and $y P_{3} 0, \alpha$ violates the priority of student 3 (D3.2). Furthermore, since $1 \succ_{y} 3$ and $(y, y) P_{c_{1}}(x, y), \beta$ violates the priority of couple $c_{1}(\mathrm{D} 3.3, \mathrm{a} .1)$.

Example 5.5 ( $p t$-acyclic priorities and no fair and efficient allocation II) Let $N=\{1,2,3,4\}$ be such that $C=\left\{c_{1}=(1,2), c_{2}=(3,4)\right\}$; and $X=\{x, y\}$ with $q_{x}=3$ and $q_{y}=1$. Let $\succ$ be such that $1 \succ_{x} 3 \succ_{x} \quad 2 \succ_{x} \quad 4$ and $2 \succ_{y}$ $1 \succ_{y} 4 \succ_{y}$ 3. Note that $\succ$ is $p t$-acyclic. Consider $R_{c_{1}}, R_{c_{2}} \in \mathcal{R C}$ such that $(x, x) P_{c_{1}}(y, x) P_{c_{1}}(0,0) P_{c_{1}} \ldots$ and $(x, x) P_{c_{2}}(y, x) P_{c_{2}}(0,0) P_{c_{2}} \ldots$, where the "tails" of $R_{c_{1}}$ and $R_{c_{2}}$, denoted by "...", can be any fixed linear order of the remaining position type assignments. The only efficient allocations for this placement problem $R=\left(R_{c_{1}}, R_{c_{2}}\right)$ are $\alpha=((x, x),(y, x))$ and $\beta=((y, x),(x, x))$. Since $3 \succ_{x} 2$ and $(x, x) P_{c_{2}}(y, x), \alpha$ violates the priority of couple $c_{2}$ (D3.3, a.1). Furthermore, since $1 \succ_{x} 3$ and $(x, x) P_{c_{1}}(y, x), \beta$ violates the priority of couple $c_{1}$ (D3.3, a.1).

Examples 5.4 and 5.5 suggest that the reason for the incompatibility of fairness and efficiency is that a couple is weakly priority-separated (D4.1). The following example demonstrates that excluding weak priority-separation of all couples does not guarantee the existence of a fair and efficient allocation.

Example 5.6 ( $p t$-acyclic priorities and no fair and efficient allocation III) Let $N=\{1,2,3,4\}$ be such that $C=\left\{c_{1}=(1,2), c_{2}=(3,4)\right\}$; and $X=\{x, y\}$ with 
$q_{x}=q_{y}=2$. Let $\succ$ be such that $1 \succ_{x} 2 \succ_{x} \quad 3 \succ_{x} \quad 4$ and $3 \succ_{y} 4 \succ_{y} 1 \succ_{y} 2$. Note that $\succ$ is $p t$-acyclic and no couple is weakly priority-separated. Consider $R_{c_{1}}, R_{c_{2}} \in \mathcal{R C}$ such that $(y, y) P_{c_{1}}(x, x) P_{c_{1}}(x, 0) P_{c_{1}}(0,0) P_{c_{1}} \ldots$ and $(x, y) P_{c_{2}}(x, 0) P_{c_{2}}(0,0) P_{c_{2}} \ldots$, where the "tails" of $R_{c_{1}}$ and $R_{c_{2}}$, denoted by “...", can be any fixed linear order of the remaining position type assignments. The only efficient allocations for this placement problem $R=\left(R_{c_{1}}, R_{c_{2}}\right)$ are $\alpha=((y, y),(x, 0))$ and $\beta=((x, 0),(x, y))$. Since $4 \succ_{y} 1$ and $(x, y) P_{c_{2}}(x, 0), \alpha$ violates the priority of couple $c_{2}$ (D3.3, a.2). Furthermore, since $2 \succ_{x} 3$ and $(x, x) P_{c_{1}}(x, 0), \beta$ violates the priority of couple $c_{1}(\mathrm{D} 3.3$, a.2).

Next, we strengthen $p t$-acyclicity in order to prevent incompatibilities as described in Examples 5.4, 5.5, and 5.6. We impose a restriction on $p t$-acyclic priority structures that will allow us to extend the DB placement mechanism to the general case with arbitrary quotas. For that purpose, we introduce the notion of strong acyclicity of the partition induced by a priority structure $\succ$. First, (i) members of a couple $c=(i, j)$ either share the same level of priority or they do not share the level of priority with any other student(s), but they have consecutive levels of priority. Second, (ii) if student $i$ has a higher level of priority than student $j$, then student $i$ has a higher priority for all position types than student $j$.

Definition 5.6 (Strongly $p t$-acyclic partition $\mathcal{S}$ induced by $\succ$ ) Let $\succ$ be a pt-acyclic priority structure and $\mathcal{S} \equiv\left\{S_{1}, \ldots, S_{p}\right\}$ be the partition (of $N$ ) induced by $\succ$. Partition $\mathcal{S}$ is strongly $p t$-acyclic if

(i) for each couple $c=(i, j)$ there is $k \in\{1, \ldots, p\}$ with $S_{k}=\{i, j\}$ or $S_{k} \cup S_{k+1}=$ $\{i, j\}$, and

(ii) for all $k, k^{\prime} \in\{1, \ldots, p\}$ such that $k<k^{\prime}$ : if $i \in S_{l}$ and $j \in S_{k^{\prime}}$, then for all $x \in X, i \succ_{x} j$.

In Example 5.4, the partition induced by $\succ$ equals $S_{1}=\{1\}$ and $S_{2}=\{2,3\}$, violating (i) in Definition 5.6. In Example 5.5 the partition induced by $\succ$ equals $S_{1}=\{1,2\}$ and $S_{2}=\{3,4\}$, violating (ii) in Definition 5.6. In Example 5.6 the partition induced by $\succ$ equals $S_{1}=\{1,3\}$ and $S_{2}=\{2,4\}$, violating (i) and (ii) in Definition 5.6.

Examples 5.4 and 5.5 demonstrate that conditions (i) and (ii) in the definition of a strong $p t$-acyclic partition are not vacuous. Lemmas 5.1 and 5.2, however, show that when all quotas are equal to one, any $p t$-acyclic priority structure induces a strongly $p t$-acyclic partition.

We will use the following notation to discuss implications of a strongly $p t$-acyclic partition. Let $c=(i, j) \in C, c^{\prime}=\left(i^{\prime}, j^{\prime}\right) \in C$, and $x \in X$. Denote $c \succ_{x} c^{\prime}$ if and only if $\left[i \succ_{x} i^{\prime}, i \succ_{x} j^{\prime}, j \succ_{x} i^{\prime}\right.$, and $\left.j \succ_{x} j^{\prime}\right]$. Furthermore, denote $c \succ c^{\prime}$ if and only if for all $x \in X, c \succ_{x} c^{\prime}$. The following direct implications of a strongly $p t$-acyclic partition $\mathcal{S}$ turn out to be useful later on.

Lemma 5.3 (Implications of a strongly pt-acyclic partition) Let $\succ$ be a pt-acyclic priority structure that induces a strongly pt-acyclic partition $\mathcal{S}=\left\{S_{1}, \ldots, S_{p}\right\}$. Then, 
(a) no couple is weakly priority-separated (D4.1);

(b) all couples have "the same sequence of priorities for all position types," i.e., for $c, c^{\prime} \in C$, either $c \succ c^{\prime}$ or $c^{\prime} \succ c$. We assume without loss of generality that $c_{1} \succ c_{2} \succ \ldots \succ c_{k}$;

(c) for all $k \in\{1, \ldots, p\}$, either $S_{k} \subseteq S$ or there exists $c=(i, j) \in C$ such that $S_{k}=\{i, j\}, S_{k-1} \cup S_{k}=\{i, j\}$, or $S_{k} \cup S_{k+1}=\{i, j\}$.

Before we introduce the so-called sequential (deferred acceptance) placement mechanism, we introduce the concept of a reduced placement problem and the deferred acceptance algorithm for single students.

Definition 5.7 (Reduced placement problems and the DA algorithm $\tilde{\varphi}$ ) Let $S^{\prime} \subseteq S$ and $q \geq q^{\prime} \geq 0$. For any $R \in \mathcal{R}$, define $R_{S^{\prime}} \equiv\left(R_{i}\right)_{i \in S^{\prime}}$. Then, given placement problem $R \in \mathcal{R}$, we denote by $\left(R_{S^{\prime}}, q^{\prime}\right)$ the reduced placement problem where position type quotas have been reduced to $q^{\prime}$ and only students in $S^{\prime}$ apply for positions.

For any reduced placement problem $\left(R_{S^{\prime}}, q^{\prime}\right)$ we determine the allocation $\tilde{\varphi}\left(R_{S^{\prime}}, q^{\prime}\right)$ using the deferred acceptance $(D A)$ algorithm of Gale and Shapley (1962):

At the first step every student in $N^{\prime}$ "proposes" to his/her favorite position type in $X \cup\{0\}$. For each position type $x$, the $q_{x}^{\prime}$ applicants who have the highest priority under $\succ_{x}$ (none if $q_{x}^{\prime}=0$ and all if there are fewer than $q_{x}^{\prime}$ ) are placed on the waiting list of $x$, and the others are rejected. Every student who applies for the null position is placed on its "waiting list."

At any consecutive step every newly rejected student proposes to his/her next best position type in $X \cup\{0\}$. For each position type $x$, the $q_{x}^{\prime}$ applicants who have the highest priority under $\succ_{x}$ (none if $q_{x}^{\prime}=0$ and all if there are fewer than $q_{x}^{\prime}$ ) among the new applicants and those on the waiting list are placed on the new waiting list and the others are rejected. Every student who applies for the null position is placed on its "waiting list."

The algorithm terminates when every student belongs to a waiting list. Then positions of type $x \in X \cup\{0\}$ are assigned to the students on the waiting list of $x$.

We can now use the strongly $p t$-acyclic partition $\mathcal{S}$ and the deferred acceptance algorithm for reduced placement problems to define the sequential deferred acceptance placement mechanism, sequential placement mechanism for short. Informally, it works as follows. Determine who has the highest level of priority. If a set of single students has the highest priority, then they receive their position types according to the DA algorithm. If a member of a couple has the highest level of priority or a couple shares the highest level of priority, then the couple receives its most preferred position assignment. Repeat this step with the remaining students and positions, i.e., determine who has the next highest level of priority, etc.

Given a reduced quota vector $q^{\prime}$ such that $q \geq q^{\prime} \geq 0$, a position type $x \in X$ is available if $q_{x}^{\prime} \geq 1$. By $X\left(q^{\prime}\right)$ we denote the set of all available position types at $q^{\prime}$. Let $s \in S$ and $R_{s} \in \mathcal{R S}$. Then top $\left(R_{s}, q^{\prime}\right)$ denotes the student's most preferred available position type in $X\left(q^{\prime}\right) \cup\{0\}$, i.e., $x=\operatorname{top}\left(R_{S}, q^{\prime}\right)$ if 
and only if for all $y \in X\left(q^{\prime}\right) \cup\{0\}, y \neq x, x P_{s} y$. Let $c \in C$ and $R_{c} \in \mathcal{R C}$. Then $\operatorname{top}\left(R_{c}, q^{\prime}\right)$ denotes the couple's most preferred available position type assignment in $\mathcal{X}\left(q^{\prime}\right) \equiv\left[\left(X\left(q^{\prime}\right) \cup\{0\}\right) \times\left(X\left(q^{\prime}\right) \cup\{0\}\right)\right] \backslash\left\{(x, x): q_{x}^{\prime}=1\right\}$, i.e., $\left(x, x^{\prime}\right)=\operatorname{top}\left(R_{c}, q^{\prime}\right) \in \mathcal{X}\left(q^{\prime}\right)$ if and only if for all $\left(y, y^{\prime}\right) \in \mathcal{X}\left(q^{\prime}\right),\left(y, y^{\prime}\right) \neq\left(x, x^{\prime}\right)$, $\left(x, x^{\prime}\right) P_{c}\left(y, y^{\prime}\right)$.

Given a set of students $S^{\prime} \subseteq S$ with allotments $\varphi_{S^{\prime}}(R) \equiv\left(\varphi_{i}(R)\right)_{i \in S^{\prime}}$, assume that $q^{\prime}$ is a (reduced) quota vector for which the students' allotments $\varphi_{S^{\prime}}(R)$ are feasible, i.e., for all $x \in X,\left|\left\{s \in S^{\prime}: \varphi_{s}(R)=x\right\}\right| \leq q_{x}^{\prime} \leq q_{x}$. Then, for all $x \in X$ we define $q_{x}^{\prime} \backslash \varphi_{S^{\prime}}(R) \equiv q_{x}^{\prime}-\left|\left\{s \in S^{\prime}: \varphi_{s}(R)=x\right\}\right|$ and $q^{\prime} \backslash \varphi_{S^{\prime}}(R) \equiv\left(q_{x}^{\prime} \backslash \varphi_{S^{\prime}}(R)\right)_{x \in X}$. Hence, $q^{\prime} \backslash \varphi_{S^{\prime}}(R)$ denotes the reduced quota vector obtained by removing the students' allotments $\varphi_{S^{\prime}}(R)$ from the placement problem.

Given a couple $c=(i, j) \in C$ with allotment $\varphi_{c}(R)=\left(\varphi_{i}(R), \varphi_{j}(R)\right)$, assume that $q^{\prime}$ is a (reduced) quota vector for which the couple's allotment $\varphi_{c}(R)$ is feasible, i.e., for all $x \in X,\left|\left\{k \in\{i, j\}: \varphi_{k}(R)=x\right\}\right| \leq q_{x}^{\prime} \leq q_{x}$. Then, for all $x \in X$ we define $q_{x}^{\prime} \backslash \varphi_{c}(R) \equiv q_{x}^{\prime}-\left|\left\{k \in\{i, j\}: \varphi_{k}(R)=x\right\}\right|$ and $q^{\prime} \backslash \varphi_{c}(R) \equiv\left(q_{x}^{\prime} \backslash \varphi_{c}(R)\right)_{x \in X}$. Hence, $q^{\prime} \backslash \varphi_{c}(R)$ denotes the reduced quota vector obtained by removing the couple's allotment $\varphi_{c}(R)$ from the placement problem.

Definition 5.8 (The sequential placement mechanism $\varphi^{\mathcal{S}}$ ) Let $\succ$ be a pt-acyclic priority structure that induces a strongly pt-acyclic partition $\mathcal{S}=\left\{S_{1}, \ldots, S_{p}\right\}$. Given $R \in \mathcal{R}$, we calculate $\varphi^{\mathcal{S}}(R)$ as follows. Set $k=1, N^{1} \equiv N$, and $q^{1} \equiv q$. As long as $k \leq p$, do Step $\boldsymbol{k}$ :

(a) $S_{k} \subseteq S: \varphi_{S_{k}}^{\mathcal{S}}(R) \equiv \tilde{\varphi}\left(R_{S_{k}}, q^{k}\right), q^{k+1} \equiv q^{k} \backslash \varphi_{S_{k}}^{\mathcal{S}}(R), N^{k+1} \equiv N^{k} \backslash S_{k}$, and set $k=k+1$.

If a set of single students $S_{k}$ share the highest priority for all remaining available position types given by $q^{k}$, then the students' assignments are determined by applying the DA algorithm to the reduced placement problem where they are the only applicants. After the students' assignments are determined, the set of remaining available positions is described by $q^{k+1}$.

(b) $S_{k}=\{s\}, s \notin S$, and $S_{k} \cup S_{k+1}=\left\{s, s^{\prime}\right\}$ such that $c=\left(s, s^{\prime}\right) \in C$ or $c=\left(s^{\prime}, s\right) \in$ $C: \varphi_{c}^{\mathcal{S}}(R) \equiv \operatorname{top}\left(R_{c}, q^{k}\right), q^{k+2} \equiv q^{k} \backslash \varphi_{c}^{\mathcal{S}}(R), N^{k+2} \equiv N^{k} \backslash\{i, j\}$, and set $k=k+2$.

If a member of a couple has the highest priority for all remaining available position types given by $q^{k}$, then the couple chooses its best pair of positions in $\mathcal{X}\left(q^{k}\right)$. After the couple's assignment is determined, the set of remaining available positions is described by $q^{k+2}$.

(c) $S_{k}=\left\{s, s^{\prime}\right\}$ and $c=\left(s, s^{\prime}\right) \in C$ or $c=\left(s^{\prime}, s\right) \in C: \varphi_{c}^{\mathcal{S}}(R) \equiv \operatorname{top}\left(R_{c}, q^{k}\right)$, $q^{k+1} \equiv q^{k} \backslash \varphi_{c}^{\mathcal{S}}(R), N^{k+1} \equiv N^{k} \backslash\{i, j\}$, and set $k=k+1$.

If the members of a couple together have the highest priority for all remaining available position types given by $q^{k}$, then the couple chooses its best pair of positions in $\mathcal{X}\left(q^{k}\right)$. After the couple's assignment is determined, the set of remaining available positions is described by $q^{k+1}$.

The allocation $\varphi^{\mathcal{S}}(R)$ is obtained in at most $p$ steps.

Note that when all quotas are equal to one, the sequential placement mechanism and the DB placement mechanism are identical. 
Example 5.7 (An application of the sequential placement mechanism) Let $N=$ $\{1, \ldots, 8\}$ be such that $C=\left\{c_{1}=(1,2), c_{2}=(3,4)\right\}$ and $S=\{5,6,7,8\}$; and $X=\{x, y, z\}$ with $q_{x}=q_{y}=q_{z}=3$. We depict the $p t$-acyclic priority structure $\succ$ and preference profile $R \in \mathcal{R}$ in the two tables below.

\begin{tabular}{|c|c|c|}
\hline$\succ_{x}$ & $\succ_{y}$ & $\succ_{z}$ \\
\hline 5 & 6 & 7 \\
6 & 7 & 5 \\
7 & 5 & 6 \\
1 & 2 & 1 \\
2 & 1 & 2 \\
3 & 3 & 3 \\
4 & 4 & 4 \\
8 & 8 & 8 \\
\hline
\end{tabular}

\begin{tabular}{|c|c|c|c|c|c|}
\hline$R_{c_{1}}$ & $R_{c_{2}}$ & $R_{5}$ & $R_{6}$ & $R_{7}$ & $R_{8}$ \\
\hline$(x, x)$ & $(x, x)$ & $y$ & $x$ & $x$ & $x$ \\
$(z, x)$ & $(z, x)$ & $x$ & $y$ & $y$ & $z$ \\
$(x, z)$ & $(x, z)$ & $z$ & $z$ & $z$ & 0 \\
$\cdots$ & $(z, z)$ & 0 & 0 & 0 & $y$ \\
& $\cdots$ & & & & \\
\hline
\end{tabular}

Priority structure $\succ$ induces the strongly $p t$-acyclic partition $S=\left\{S_{1}, S_{2}, S_{3}, S_{4}, S_{5}\right\}$ with $S_{1}=\{5,6,7\}, S_{2}=\{1,2\}, S_{3}=\{3\}, S_{4}=\{4\}$, and $S_{5}=\{8\}$. Note that $S_{4} \cup S_{5}=\{3,4\}$ consists of couple $c_{2}$. We now calculate $\varphi^{\mathcal{S}}(R)$. Recall that $N^{1}=N$ and $q^{1} \equiv q=\left(q_{x}, q_{y}, q_{z}\right)=(3,3,3)$.

Step 1: $S_{1}=\{5,6,7\} \subseteq S: \varphi_{5}^{\mathcal{S}}(R)=y, \varphi_{6}^{\mathcal{S}}(R)=x, \varphi_{7}^{\mathcal{S}}(R)=x, N^{2}=\{1,2,3,4,8\}$, and $q^{2}=\left(q_{x}^{2}, q_{y}^{2}, q_{z}^{2}\right)=(1,2,3)$.

Step 2: $S_{2}=\{1,2\}$ and $c_{1}=(1,2) \in C: \varphi_{c_{1}}^{\mathcal{S}}(R)=(z, x), N^{3}=\{3,4,8\}$, and $q^{3}=\left(q_{x}^{3}, q_{y}^{3}, q_{z}^{3}\right)=(0,2,2)$.

Step 3: $S_{3} \cup S_{4}=\{3,4\}$ and $c_{2}=(3,4) \in C: \varphi_{c_{2}}^{\mathcal{S}}(R)=(z, z), N^{5}=\{8\}$, and $q^{5}=\left(q_{x}^{5}, q_{y}^{5}, q_{z}^{5}\right)=(0,2,0)$.

Step 5: $S_{5}=\{8\} \subseteq S: \varphi_{8}^{\mathcal{S}}(R)=0$.

Hence, $\varphi^{\mathcal{S}}(R)=(z, x, z, z, y, x, x, 0)$.

Our next result is that for $p t$-acyclic priority structures that induce strongly $p t$-acyclic partitions the sequential placement mechanism is fair and efficient.

Theorem 5.3 (General quotas: sufficiency of a strongly $p t$-acyclic partition) Let $\succ$ be a pt-acyclic priority structure that induces a strongly pt-acyclic partition. Then, the sequential placement mechanism $\varphi^{\mathcal{S}}$ is fair and efficient.

Remark 5.2 (Responsiveness in student placement and two-sided matching) The student placement model we consider and two-sided matching markets (see Roth and Sotomayor 1990) are closely related. In our context of student placement, we consider students' preferences and a priority structure as inputs and focus on fairness and efficiency. By contrast, a two-sided matching problem also consists of students' preferences, but priorities over position types are replaced by preferences over students or sets of students of the institutions that offer the position types (e.g., universities, firms, or hospitals). An important property for two-sided matching is stability: loosely speaking, an outcome or matching is stable if there are no students (couples) and no institutions that are not matched with each other, but in fact would prefer to be. A key result (among 
many other results) for two-sided matching problems is that under the appropriate substitutability or responsiveness condition stable matchings always exist; see for instance Roth (1985, many-to-one matching without money), Kelso and Crawford (1982, many-to-one matching with money), Alkan and Gale (2003, many-to-many schedule matching), Klaus and Klijn (2005, many-to-one matching with couples), and Hatfield and Milgrom (2005, two-sided matching with contracts). More specifically, whenever couples with non-responsive preferences are present in a two-sided matching market, a stable outcome may not exist; see Roth (1984) and Klaus and Klijn (2005). Klaus and Klijn (2005, 2007) show that indeed responsiveness of couples' preferences is often a necessary condition for their results.

Because of the important role that responsiveness plays in two-sided matching markets, one may wonder if one could obtain stronger results for the student placement problems with couples if couples' preferences are restricted to always be responsive. However, since all proofs are designed in such a way that they also apply if couples' preferences are responsive, requiring that all couples' preferences are responsive will not change any of our results.

\section{An open problem: necessity and uniqueness}

We have shown that $p t$-acyclicity of the priority structure is a necessary and, in combination with strong $p t$-acyclicity of the induced partition, a sufficient condition for the existence of a fair and efficient placement mechanism. The determination of further necessary conditions is an open problem. Consequently, we were also not able to address the question of uniqueness for general placement problems.

Acknowledgments We thank Howard Petith, William Thomson, and two anonymous referees for helpful comments and suggestions. B. Klaus's and F. Klijn's research was supported by Ramón y Cajal contracts of the Spanish Ministerio de Ciencia y Tecnología. The work of the authors was partially supported by the Spanish Plan Nacional I+D+I (BEC2002-02130, SEJ2005-01690) and by the Generalitat de Catalunya (SGR2005-00626 and the Barcelona Economics Program of CREA).

\section{Appendix: proofs}

Proof of Theorem 4.1 (a) Assume $\succ$ violates acyclicity. Thus, there exists a cycle, i.e., there exist distinct $x, y \in X$ and $i, j, k \in N$ such that the following two conditions are satisfied:

cycle condition $i \succ_{x} j \succ_{x} k$ and $k \succ_{y} i$ and c-scarcity condition there exist disjoint sets $N_{x}, N_{y} \subseteq N \backslash\{i, j, k\}$ (possibly $N_{x}=$ $\emptyset$ or $\left.N_{y}=\emptyset\right)$ such that $N_{x} \subseteq\left\{l \in N: l \succ_{x} j\right\}, N_{y} \subseteq\left\{l \in N: l \succ_{y} i\right\},\left|N_{x}\right|=q_{x}-1$, and $\left|N_{y}\right|=q_{y}-1$.

We now construct a preference profile $R \in \mathcal{R}$. We complete the proof by showing that no fair and efficient mechanism exists.

First, we specify preferences in $\mathcal{R S}$ for all students $s \in N$. Let $N_{0} \equiv$ $N \backslash\left[\{i, j, k\} \cup N_{x} \cup N_{y}\right]$.

(SP) Students' preferences: Let $s \in N$ and $R_{s} \in \mathcal{R S}$ be such that 
if $s \in N_{0}$, then $0 P_{s} \ldots$,

if $s \in N_{x} \cup\{j\}$, then $x P_{s} 0 P_{s} \ldots$,

if $s \in N_{y}$, then $y P_{s} 0 P_{s} \ldots$,

if $s=i$, then $y P_{s} \times P_{s} 0 P_{s} \ldots$, and

if $s=k$, then $x P_{s} y P_{s} 0 P_{s} \ldots$,

where the "tail" of any of the above preference relations, denoted by "...", can be any fixed linear order of the remaining position types.

Second, using the above specification of preferences for students, we specify responsive preferences for all possible couples.

(CP) Couples' preferences: Let $c=(l, m) \in C$. We specify $R_{c} \in \mathcal{R} \mathcal{R C}$ such that couple $c$ 's preferences are responsive with respect to associated individual preferences $R_{l}$ and $R_{m}$ that are as above. Let $R_{c} \in \mathcal{R} \mathcal{R C}$ be such that ${ }^{6}$

if $l, m \in N_{0}$, then $(0,0) P_{c} \ldots$,

if $l \in N_{0}$ and $m \in N_{x} \cup\{j\}$, then $(0, x) P_{c}(0,0) P_{c} \ldots$,

if $l \in N_{0}$ and $m \in N_{y}$, then $(0, y) P_{c}(0,0) P_{c} \ldots$,

if $l \in N_{0}$ and $m=i$, then $(0, y) P_{c}(0, x) P_{c}(0,0) P_{c} \ldots$,

if $l \in N_{0}$ and $m=k$, then $(0, x) P_{c}(0, y) P_{c}(0,0) P_{c} \ldots$,

if $l \in N_{x} \cup\{j\}$ and $m \in N_{0}$, then $(x, 0) P_{c}(0,0) P_{c} \ldots$,

if $l \in N_{x}$ and $m \in N_{x} \cup\{j\}$, then $(x, x) P_{c}(x, 0) P_{c}(0, x) P_{c}(0,0) P_{c} \ldots$,

if $l=j$ and $m \in N_{x}$, then $(x, x) P_{c}(0, x) P_{c}(x, 0) P_{c}(0,0) P_{c} \ldots$,

if $l \in N_{x} \cup\{j\}$ and $m \in N_{y}$, then $(x, y) P_{c}(0, y) P_{c}(x, 0) P_{c}(0,0) P_{c} \ldots$,

if $l \in N_{x}$ and $m=i$, then $(x, y) P_{c}(x, x) P_{c}(x, 0) P_{c}(0, y) P_{c}(0, x) P_{c}(0,0) P_{c} \ldots$, if $l=j$ and $m=i$, then $(x, y) P_{c}(0, y) P_{c}(x, x) P_{c}(0, x) P_{c}(x, 0) P_{c}(0,0) P_{c} \ldots$, if $l \in N_{x} \cup\{j\}$ and $m=k$, then $(x, x) P_{c}(x, y) P_{c}(x, 0) P_{c}(0, x) P_{c}(0, y) P_{c}(0,0) P_{c} \ldots$, if $l \in N_{y}$ and $m \in N_{0}$, then $(y, 0) P_{c}(0,0) P_{c} \ldots$,

if $l \in N_{y}$ and $m \in N_{x} \cup\{j\}$, then $(y, x) P_{c}(y, 0) P_{c}(0, x) P_{c}(0,0) P_{c} \ldots$,

if $l, m \in N_{y}$, then $(y, y) P_{c}(y, 0) P_{c}(0, y) P_{c}(0,0) P_{c} \ldots$,

if $l \in N_{y}$ and $m=i$, then $(y, y) P_{c}(y, x) P_{c}(y, 0) P_{c}(0, y) P_{c}(0, x) P_{c}(0,0) P_{c} \ldots$, if $l \in N_{y}$ and $m=k$, then $(y, x) P_{c}(y, y) P_{c}(y, 0) P_{c}(0, x) P_{c}(0, y) P_{c}(0,0) P_{c} \ldots$, if $l=i$ and $m \in N_{0}$, then $(y, 0) P_{c}(x, 0) P_{c}(0,0) P_{c} \ldots$,

if $l=i$ and $m \in N_{x}$, then $(y, x) P_{c}(x, x) P_{c}(0, x) P_{c}(y, 0) P_{c}(x, 0) P_{c}(0,0) P_{c} \ldots$, if $l=i$ and $m=j$, then $(y, x) P_{c}(y, 0) P_{c}(x, x) P_{c}(x, 0) P_{c}(0, x) P_{c}(0,0) P_{c} \ldots$, if $l=i$ and $m \in N_{y}$, then $(y, y) P_{c}(x, y) P_{c}(0, y) P_{c}(y, 0) P_{c}(x, 0) P_{c}(0,0) P_{c} \ldots$, if $l=i$ and $m=k$,

then $(y, x) P_{c}(y, y) P_{c}(x, x) P_{c}(x, y) P_{c}(y, 0) P_{c}(x, 0) P_{c}(0, x) P_{c}(0, y) P_{c}(0,0) P_{c} \ldots$, if $l=k$ and $m \in N_{0}$, then $(x, 0) P_{c}(y, 0) P_{c}(0,0) P_{c} \ldots$,

if $l=k$ and $m \in N_{x} \cup\{j\}$, then $(x, x) P_{c}(y, x) P_{c}(0, x) P_{c}(x, 0) P_{c}(y, 0) P_{c}(0,0) P_{c} \ldots$, if $l=k$ and $m \in N_{y}$, then $(x, y) P_{c}(y, y) P_{c}(0, y) P_{c}(x, 0) P_{c}(y, 0) P_{c}(0,0) P_{c} \ldots$, and if $l=k$ and $m=i$,

then $(x, y) P_{c}(y, y) P_{c}(x, x) P_{c}(y, x) P_{c}(0, y) P_{c}(0, x) P_{c}(x, 0) P_{c}(y, 0) P_{c}(0,0) P_{c} \ldots$,

${ }^{6}$ If $q_{x}=1$, then delete $(x, x)$ from all couples' preferences specified below. If $q_{y}=1$, then delete $(y, y)$ from all couples' preferences specified below. 
where the "tail" of any of the above preference relations, denoted by "...", can be any fixed linear order of the remaining position type assignments that complies with the responsiveness requirement induced by the associated individual preferences.

Finally, we define $R \in \mathcal{R}$ such that single students have preferences as specified in (SP) and couples have preferences as specified in (CP). We now complete the proof by showing that there is no fair and efficient mechanism $\varphi$. Suppose to the contrary there is such a mechanism.

Step 1: We prove that for all $l \in N_{0}, \varphi_{l}(R)=0$.

Let $l \in N_{0} \cap S$. By efficiency, $\varphi_{l}(R)=0$.

Let $l \in N_{0} \backslash S$ and assume that $\varphi_{l}(R) \neq 0$ and $c=(l, m)$ (for $c=(m, l)$ interchange the roles of $l$ and $m$ ). Recall that the couple's preferences $R_{c}$ are responsive with respect to $R_{l}, R_{m} \in \mathcal{R S}$. Thus, $0 P_{l} \varphi_{l}(R)$ implies $\left(0, \varphi_{m}(R)\right) P_{c}$ $\left(\varphi_{l}(R), \varphi_{m}(R)\right)$. Since the null position is freely available, this contradicts efficiency.

Step 2: We prove that for all $l \in N_{x}, \varphi_{l}(R)=x$.

Let $l \in N_{x}$ and assume that $\varphi_{l}(R) \neq x$. Then, by efficiency, the definition of single students' preferences, and responsiveness of couples' preferences, $\varphi_{l}(R)=0$ and all position types $x$ have to be assigned to students in $\left[N_{x} \cup\{i, j, k\}\right] \backslash\{l\}$. Hence, there exist (at least) two distinct students in $\{i, j, k\}$ whose allotment equals $x$. Thus,

$$
\varphi_{j}(R)=x \text { or } \varphi_{k}(R)=x .
$$

Let $l \in N_{x} \cap S$. Since $l \succ_{x} j \succ_{x} k$, (1) violates student $l$ 's priority (D3.2).

Let $l \in N_{x} \backslash S$ and $c=(l, m)$ (for $c=(m, l)$ interchange the roles of $l$ and $m$ ).

If $m \in N \backslash\{j, k\}$, then by responsiveness of $R_{c},\left(x, \varphi_{m}(R)\right) P_{c}\left(\varphi_{l}(R), \varphi_{m}(R)\right)$. (Note that because there are at least two distinct students in $\{i, j, k\}$ whose allotment equals $x, q_{x} \geq 2$ and $\left(x, \varphi_{m}(R)\right) \in \mathcal{X}$.) Since $l \succ_{x} j \succ_{x} k$, (1) violates couple $c$ 's priority (D3.3, a.1).

If $m \in\{j, k\}$ and $\varphi_{m}(R)=x$, then by our definition of $R_{c},(x, 0) P_{c}(0, x)=$ $\left(\varphi_{l}(R), \varphi_{m}(R)\right)$. Since $l \succ_{x} m,(1)$ violates couple $c$ 's priority (D3.3, b.1).

If $m \in\{j, k\}$ and $\varphi_{m}(R) \neq x$, then by responsiveness of $R_{c},\left(x, \varphi_{m}(R)\right) P_{c}$ $\left(\varphi_{l}(R), \varphi_{m}(R)\right)$. Let $\left\{m, m^{\prime}\right\}=\{j, k\}$. Then, by $(1), \varphi_{m^{\prime}}(R)=x$. Since $l \succ_{x} m^{\prime}$, $\varphi_{m^{\prime}}(R)=x$ violates couple $c$ 's priority (D3.3, a.1).

Step 3: We prove that for all $l \in N_{y}, \varphi_{l}(R)=y$.

Let $l \in N_{y}$ and assume that $\varphi_{l}(R) \neq y$. Then, by efficiency, the definition of single students' preferences, and responsiveness of couples' preferences, $\varphi_{l}(R)=0$ and all position types $y$ have to be assigned to students in $\left[N_{y} \cup\{i, k\}\right] \backslash\{l\}$. Hence, $\varphi_{k}(R)=y$ and

$$
\varphi_{i}(R)=y .
$$

Let $l \in N_{y} \cap S$. Since $l \succ_{y} i$,(2) violates student $l$ 's priority (D3.2).

Let $l \in N_{y} \backslash S$ and $c=(l, m)$ (for $c=(m, l)$ interchange the roles of $l$ and $m$ ).

If $m \in N \backslash\{i\}$, then by responsiveness of $R_{c},\left(y, \varphi_{m}(R)\right) P_{c}\left(\varphi_{l}(R), \varphi_{m}(R)\right)$. (Note that because $\varphi_{k}(R)=\varphi_{i}(R)=y, q_{y} \geq 2$ and $\left(y, \varphi_{m}(R)\right) \in \mathcal{X}$.) Since $l \succ_{y} i,(2)$ violates couple $c$ 's priority (D3.3, a.1). 
If $m=i$, then by our definition of $R_{c},(y, 0) P_{c}(0, y)=\left(\varphi_{l}(R), \varphi_{m}(R)\right)$. Since $l \succ_{y} m,(2)$ violates couple $c$ 's priority (D3.3, b.1).

Note that now only one position of type $x$ and one position of type $y$ is "left to be assigned" to the students in $\{i, j, k\}$.

Step 4: We prove that $\varphi_{j}(R)=x$.

Suppose that $\varphi_{j}(R) \neq x$. Then, by efficiency, the definition of single students' preferences, and responsiveness of couples' preferences, $\varphi_{j}(R)=0, \varphi_{i}(R)=y$, and $\varphi_{k}(R)=x$.

Suppose $j \in S$. Since $j \succ_{x} k, \varphi_{k}(R)=x$ violates student $j$ 's priority (D3.2).

So suppose $j \in N \backslash S$ and let $c=(j, m)$ (for $c=(m, j)$ interchange the roles of $j$ and $m$ ).

If $m \in N \backslash\{k\}$, then by responsiveness of $R_{c},\left(x, \varphi_{m}(R)\right) P_{c}\left(\varphi_{j}(R), \varphi_{m}(R)\right)$. (Note that $\left(x, \varphi_{m}(R)\right) \in \mathcal{X}$. If not, then $q_{x}=1$ and $\varphi_{m}(R)=x$, contradicting $m \neq k$.) Since $j \succ_{x} k, \varphi_{k}(R)=x$ violates couple $c$ 's priority (D3.3, a.1).

If $m=k$, then by our definition of $R_{c},(x, 0) P_{c}(0, x)=\left(\varphi_{j}(R), \varphi_{m}(R)\right)$. Since $j \succ_{x} m, \varphi_{k}(R)=x$ violates couple $c$ 's priority (D3.3, b.1).

Now only one position of type $y$ is "left to be assigned" to either student $i$ or $k$. Hence, by efficiency, the definition of single students' preferences, and responsiveness of couples' preferences, either $\left[\varphi_{i}(R)=y\right.$ and $\left.\varphi_{k}(R)=0\right]$ or $\left[\varphi_{i}(R)=0\right.$ and $\left.\varphi_{k}(R)=y\right]$.

Step 5: We obtain a contradiction.

Suppose $i \in S$. Suppose $\varphi_{i}(R)=y$ and $\varphi_{k}(R)=0$. Recall that $k \succ_{y} i$.

If $k \in S, \varphi_{i}(R)=y$ violates student $k$ 's priority (D3.2).

If $k \in N \backslash S$, then let $c=(k, m)$ (for $c=(m, k)$ interchange the roles of $k$ and $m)$. By responsiveness of $R_{c},\left(y, \varphi_{m}(R)\right) P_{c}\left(\varphi_{k}(R), \varphi_{m}(R)\right)$. (Note that $\left(y, \varphi_{m}(R)\right) \in \mathcal{X}$. If not, then $q_{y}=1$ and $\varphi_{m}(R)=y$, contradicting $m \neq$ i.) Since $k \succ_{y} i, \varphi_{i}(R)=y$ violates couple $c$ 's priority (D3.3, a.1). Hence, $\varphi_{i}(R)=0$ and $\varphi_{k}(R)=y$.

Note that $x P_{i} \varphi_{i}(R)$. Since $i \succ_{x} j, \varphi_{j}(R)=x$ violates student $i$ 's priority (D3.2).

So suppose $i \in N \backslash S$ and let $c=(i, m)$ (for $c=(m, i)$ interchange the roles of $i$ and $m$ ). If $m=k$, then by $(y, 0) P_{c}(0, y)$ and efficiency, $\varphi_{i}(R)=y$ and $\varphi_{k}(R)=0$. Thus, by the construction of preference relation $R_{c},(x, y) P_{c}\left(\varphi_{i}(R), \varphi_{k}(R)\right)$. Since $i \succ_{x} j$ and $k \succ_{y} i, \varphi_{j}(R)=x$ and $\varphi_{i}(R)=y$ violate couple $c$ 's priority (D3.3,c). Hence, $m \neq k$. Suppose $\varphi_{i}(R)=y$ and $\varphi_{k}(R)=0$. Recall that $k \succ_{y} i$.

If $k \in S, \varphi_{i}(R)=y$ violates student $k$ 's priority (D3.2).

If $k \in N \backslash S$, then let $\bar{c}=(k, \bar{m})$ (for $\bar{c}=(\bar{m}, k)$ interchange the roles of $k$ and $\bar{m})$. By responsiveness of $R_{\bar{c}},\left(y, \varphi_{\bar{m}}(R)\right) P_{\bar{c}}\left(\varphi_{k}(R), \varphi_{\bar{m}}(R)\right)$. (Note that $\left(y, \varphi_{\bar{m}}(R)\right) \in \mathcal{X}$. If not, then $q_{y}=1$ and $\varphi_{\bar{m}}(R)=y$, contradicting $\bar{m} \neq i$.) Since $k \succ_{y} i, \varphi_{i}(R)=y$ violates couple $\bar{c}$ 's priority (D3.3, a.1). Hence, $\varphi_{i}(R)=0$ and $\varphi_{k}(R)=y$.

If $m \in N \backslash\{j, k\}$, then by responsiveness of $R_{c},\left(x, \varphi_{m}(R)\right) P_{c}\left(\varphi_{i}(R), \varphi_{m}(R)\right)$. (Note that $\left(x, \varphi_{m}(R)\right) \in \mathcal{X}$. If not, then $q_{x}=1$ and $\varphi_{m}(R)=x$, contradicting $m \neq j$.) Since $i \succ_{x} j, \varphi_{j}(R)=x$ violates couple $c$ 's priority (D3.3, a.1).

If $m=j$, then by Step $4, \varphi_{c}(R)=(0, x)$. Since $(x, 0) P_{c}(0, x)=\varphi_{c}(R), \varphi(R)$ is not an efficient allocation. Alternatively, since $i \succ_{x} j, \varphi_{j}(R)=x$ violates couple $c$ 's priority (D3.3, b.1). 
(b) Let $N=\{1,2,3\}$ be such that $C=\{(1,2)\}$ and $S=\{3\}, X=\{x\}$, and $q_{x}=1$. Let $\succ$ be such that $1 \succ_{x} 3 \succ_{x}$ 2. Since $|X|=1, \succ$ is acyclic. Assume that $\varphi$ is fair and efficient. Consider $R=\left(R_{c}, R_{3}\right) \in \mathcal{R}$ such that $(0, x) P_{c}(x, 0) P_{c}(0,0)$ and $x P_{3} 0$. Note that $R_{c} \in \mathcal{R} \mathcal{R C}$.

If $\varphi_{3}(R)=x$, then $\varphi_{c}(R)=(0,0)$. Since $1 \succ_{x} 3,(x, 0) P_{c}(0,0)=\varphi_{c}(R)$ violates couple $c$ 's priority (D3.3, a.1).

If $\varphi_{3}(R)=0$, by efficiency, $\varphi_{c}(R)=(0, x)$. Since $3 \succ_{x} \quad 2, x P_{3} 0=\varphi_{3}(R)$ violates student 3's priority (D3.2).

Proof of Theorem 4.2 By Theorem 4.1 (a) we already know that acyclicity is necessary for the existence of a fair and efficient placement mechanism. So assume $\succ$ violates priority-togetherness, i.e., there exists $\bar{c}=(i, j) \in C$ that is priority-separated. Without loss of generality there exist $x \in X$ and $k \in N$ such that

priority-separation condition $i \succ_{x} k \succ_{x} j$ and

ps-scarcity condition there exists a set $\bar{N}_{x} \subseteq N \backslash\{i, j, k\}$ (possibly $\bar{N}_{x}=\emptyset$ ) such that $\bar{N}_{x} \subseteq\left\{l \in N: l \succ_{x} j\right\}$ and $\left|\bar{N}_{x}\right|=q_{x}-1$.

We now construct a preference profile $R \in \mathcal{R}$. We complete the proof by showing that no fair and efficient mechanism exists.

First, we specify preferences in $\mathcal{R S}$ for all students $s \in N$. Let $\bar{N}_{0} \equiv$ $N \backslash\left[\{i, j, k\} \cup \bar{N}_{x}\right]$.

(SP) Students' preferences: Let $s \in N$ and $R_{s} \in \mathcal{R S}$ be such that

if $s \in \bar{N}_{0}$, then $0 P_{s} \ldots$,

if $s \in \bar{N}_{x} \cup\{i, j, k\}$, then $x P_{s} 0 P_{s} \ldots$,

where the "tail" of any of the above preference relations, denoted by "...", can be any fixed linear order of the remaining position types.

Second, using the above specification of preferences for students, we specify responsive preferences for all possible couples.

(CP) Couples' preferences: Let $c=(l, m) \in C \backslash\{\bar{c}\}$. We specify $R_{c} \in \mathcal{R} \mathcal{R C}$ such that couple $c$ 's preferences are responsive with respect to associated individual preferences $R_{l}$ and $R_{m}$ as above. Without loss of generality, assume that $q_{x}>1$. Let $R_{c} \in \mathcal{R} \mathcal{R C}$ be such that ${ }^{7}$

if $l, m \in \bar{N}_{0}$, then $(0,0) P_{c} \ldots$,

if $l \in \bar{N}_{0}$ and $m \in \bar{N}_{x} \cup\{k\}$, then $(0, x) P_{c}(0,0) P_{c} \ldots$,

if $l \in \bar{N}_{x} \cup\{k\}$ and $m \in \bar{N}_{0}$, then $(x, 0) P_{c}(0,0) P_{c} \ldots$,

if $l, m \in \bar{N}_{x} \cup\{k\}$, then $(x, x) P_{c}(0, x) P_{c}(x, 0) P_{c}(0,0) P_{c} \ldots$,

and in addition

$(x, x) P_{\bar{c}}(0, x) P_{\bar{c}}(x, 0) P_{\bar{c}}(0,0) P_{\bar{c}} \ldots$,

where the "tail" of any of the above preference relations, denoted by “...", can be any fixed linear order of the remaining position type assignments that complies with the responsiveness requirement induced by the associated individual preferences.

7 If $q_{x}=1$, then delete $(x, x)$ from all couples' preferences specified below. 
Finally, we define $R \in \mathcal{R}$ such that single students have preferences as specified in (SP) and couples have preferences as specified in (CP). We now complete the proof by showing that there is no fair and efficient mechanism $\varphi$. Suppose to the contrary there is such a mechanism.

Step 1: We prove that for all $l \in \bar{N}_{0}, \varphi_{l}(R)=0$.

Let $l \in \bar{N}_{0} \cap S$. By efficiency, $\varphi_{l}(R)=0$.

Let $l \in \bar{N}_{0} \backslash S$ and assume that $\varphi_{l}(R) \neq 0$ and $c=(l, m)$ (for $c=(m, l)$ interchange the roles of $l$ and $m$ ). Recall that the couple's preferences $R_{c}$ are responsive with respect to $R_{l} \in \mathcal{R S}$ and $R_{m} \in \mathcal{R S}$. Thus, $0 P_{l} \varphi_{l}(R)$ implies $\left(0, \varphi_{m}(R)\right) P_{c}\left(\varphi_{l}(R), \varphi_{m}(R)\right)$. Since the null position is freely available, this contradicts efficiency.

Step 2: We prove that for all $l \in \bar{N}_{x} \cup\{k\}, \varphi_{l}(R)=x$.

Let $l \in \bar{N}_{x} \cup\{k\}$ and assume that $\varphi_{l}(R) \neq x$. Then, by efficiency, the definition of single students' preferences, and responsiveness of couples' preferences, $\varphi_{l}(R)=0$ and all position types $x$ have to be assigned to students in $\left[\bar{N}_{x} \cup\{i, j, k\}\right] \backslash\{l\}$. Hence,

$$
\varphi_{i}(R)=x \text { or } \varphi_{j}(R)=x .
$$

Assume that $\varphi_{j}(R)=x$.

Let $l \in\left[\bar{N}_{x} \cup\{k\}\right] \cap S$. Since $l \succ_{x} j, \varphi_{j}(R)=x$ violates student $l$ 's priority (D3.2).

Let $l \in\left[\bar{N}_{x} \cup\{k\}\right] \backslash S$ and $c=(l, m)$ (for $c=(m, l)$ interchange the roles of $l$ and $m$ ).

Then, by responsiveness of $R_{c},\left(x, \varphi_{m}(R)\right) P_{c}\left(\varphi_{l}(R), \varphi_{m}(R)\right)$. (Note that $\left(x, \varphi_{m}(R)\right) \in \mathcal{X}$. If not, then $q_{x}=1$ and $\varphi_{m}(R)=x$. Hence, $m=j$. But then $c=\bar{c}$, i.e., $l=i$. Hence, $i=l \in \bar{N}_{x} \cup\{k\}$, a contradiction.) Since $l \succ_{x} j, \varphi_{j}(R)=x$ violates couple $c$ 's priority (D3.3, a.1).

Hence, $\varphi_{j}(R)=0$ and by $(3), \varphi_{\bar{c}}(R)=(x, 0)$. Since $(0, x) P_{\bar{c}}(x, 0)=\varphi_{\bar{c}}(R)$, $\varphi(R)$ is not an efficient allocation.

Step 3: We obtain a contradiction.

Step 2 implies that $\varphi_{k}(R)=x$ and $\varphi_{\bar{c}}(R)=(0,0)$. Since $i \succ_{x} k,(x, 0) P_{\bar{c}}(0,0)=$ $\varphi_{\bar{c}}(R)$ violates couple $\bar{c}$ 's priority (D3.3, a.1).

Proof of Lemma 5.1 Recall that all quotas equal one. We prove Lemma 5.1 in four steps. Steps 1 and 2 show that $p t$-acyclicity implies conditions (i) and (ii) and Steps 3 and 4 show that conditions (i) and (ii) imply $p t$-acyclicity.

Step 1: "acyclicity $\Rightarrow$ (i)"

Let $\succ$ be acyclic and $k \in\{1, \ldots, p\}$. We prove that acyclicity implies (i.a) and (i.b) for $S_{k}$.

Assume that (i.a) is violated for $S_{k}$. Suppose that $\left|S_{k}\right|>2$, e.g., there exist distinct $i_{1}, i_{2}, i_{3}$ such that $S_{k}=\left\{i_{1}, i_{2}, i_{3}, \ldots\right\} \subseteq N \backslash \bigcup_{l=1}^{k-1} S_{l}{ }^{8}$ Since $i_{1}, i_{2}, i_{3} \in S_{k}$, there exist distinct $x_{1}, x_{2}, x_{3} \in X$ such that $i_{1}=\operatorname{top}\left(x_{1}, N \backslash \bigcup_{l=1}^{k-1} S_{l}\right), i_{2}=$

8 If $k=1$, set $\bigcup_{l=1}^{k-1} S_{l}=\emptyset$. 
$\operatorname{top}\left(x_{2}, N \backslash \bigcup_{l=1}^{k-1} S_{l}\right)$, and $i_{3}=\operatorname{top}\left(x_{3}, N \backslash \bigcup_{l=1}^{k-1} S_{l}\right)$. Hence, $i_{1} \succ_{x_{1}} i_{2}, i_{1} \succ_{x_{1}} i_{3}$, $i_{2} \succ_{x_{2}} i_{1}$, and $i_{3} \succ_{x_{3}} i_{1}$. Note that either $i_{2} \succ_{x_{1}} i_{3}$ or $i_{3} \succ_{x_{1}} i_{2}$. Thus, in contradiction to acyclicity, either $\left[i_{1} \succ_{x_{1}} i_{2} \succ_{x_{1}} i_{3}\right.$ and $\left.i_{3} \succ_{x_{3}} i_{1}\right]$ or $\left[i_{1} \succ_{x_{1}} i_{3} \succ_{x_{1}} i_{2}\right.$ and $\left.i_{2} \succ_{x_{2}} i_{1}\right]$ constitutes a cycle. Hence, (i.a) for $S_{k}$ is implied by acyclicity.

Assume that (i.b) is violated for $S_{k}$. Suppose $S_{k}=\{i, j\}$ and there exists $x \in X$ such that $i=\operatorname{top}\left(x, N \backslash \bigcup_{l=1}^{k-1} S_{l}\right)$ and $j \neq \operatorname{top}\left(x, N \backslash\left[\bigcup_{l=1}^{k-1} S_{l} \cup\{i\}\right]\right)$. Thus, there exists $j^{\prime} \in N \backslash\left[\bigcup_{l=1}^{k-1} S_{l} \cup\{i, j\}\right]$ such that $j^{\prime}=\operatorname{top}\left(x, N \backslash\left[\bigcup_{l=1}^{k-1} S_{l} \cup\{i\}\right]\right)$. Hence, $i \succ_{x} j^{\prime} \succ_{x} j$. Since $j \in S_{k}$, there exists $y \in X$ such that $j=\operatorname{top}\left(y, N \backslash \bigcup_{l=1}^{k-1} S_{l}\right)$. Hence, $j \succ_{y} i$. Thus, in contradiction to acyclicity, $i \succ_{x} j^{\prime} \succ_{x} j$ and $j \succ_{y} i$ constitutes a cycle. Hence, (i.b) for $S_{k}$ is implied by acyclicity.

Step 2: "pt-acyclicity $\Rightarrow$ (ii)"

Let $\succ$ be $p t$-acyclic. Hence, $\succ$ is acyclic and by Step 1, Lemma 5.1 (i) (and therefore Lemma 5.2) applies. Assume that there exists a couple $c=(i, j) \in C$ such that (ii) is violated. Then, there exists $k \in\{1, \ldots, p\}$ such that $\left[S_{k}=\{i, m\}\right.$ for $m \in N \backslash\{i, j\}]$ or $\left[S_{k}=\{j, m\}\right.$ for $\left.m \in N \backslash\{i, j\}\right]$, or $\left[S_{k}=\{i\}\right.$ and there exists $k^{\prime} \in\{1, \ldots, p\} \backslash\{k-1, k, k+1\}$ such that $\left.S_{k^{\prime}}=\{j\}\right]$.

First, assume that $S_{k}=\{i, m\}$ for $m \in N \backslash\{i, j\}$. Let $k^{\prime} \in\{1, \ldots, p\}$ be such that $j \in S_{k^{\prime}}$.

If $k<k^{\prime}$, then by Lemma 5.2 (b), for all $x \in X, i \succ_{x} j$ and $m \succ_{x} j$. Since $i \in S_{k}$, there exists $y \in X$ such that $i=\operatorname{top}\left(y, N \backslash \bigcup_{l=1}^{k-1} S_{l}\right)$. Hence, $i \succ_{y} m$. Thus, $i \succ_{y} m \succ_{y} j$ and, in contradiction to priority-togetherness, couple $c$ is priority-separated.

If $k>k^{\prime}$, then by Lemma 5.2 (b), for all $x \in X, j \succ_{x} i$ and $j \succ_{x} m$. Since $m \in S_{k}$, there exists $y \in X$ such that $m=\operatorname{top}\left(y, N \backslash \bigcup_{l=1}^{k-1} S_{l}\right)$. Hence, $m \succ_{y} i$. Thus, $j \succ_{y} m \succ_{y} i$, and, in contradiction to priority-togetherness, couple $c$ is priority-separated.

Second, assume $S_{k}=\{j, m\}$ for $m \in N \backslash\{i, j\}$. By interchanging the roles if $i$ and $j$ in the proof above we again obtain a contradiction to priority-togetherness.

Third, assume that $S_{k}=\{i\}$ and there exists $k^{\prime} \in\{1, \ldots, p\} \backslash\{k-1, k, k+1\}$ such that $S_{k^{\prime}}=\{j\}$.

If $k<k^{\prime}$, then $k<k+1<k^{\prime}$. Let $m \in S_{k+1}$. Then, by Lemma 5.2 (b), for all $x \in X, i \succ_{x} m \succ_{x} j$, which contradicts priority-togetherness.

If $k>k^{\prime}$, then $k>k-1>k^{\prime}$. Let $m \in S_{k-1}$. Then, by Lemma 5.2 (b), for all $x \in X, j \succ_{x} m \succ_{x} i$, which contradicts priority-togetherness.

Step 3: "(i) $\Rightarrow$ acyclicity"

Let $\mathcal{S}$ satisfy (i). Then, Lemma 5.2 applies as well.

Let $i_{1}, i_{2}, i_{3} \in N$ and $x \in X$ be such that $i_{1} \succ_{x} i_{2} \succ_{x} i_{3}$. Furthermore, let $k_{1}, k_{3} \in\{1, \ldots, p\}$ be such that $i_{1} \in S_{k_{1}}$ and $i_{3} \in S_{k_{3}}$. By Lemma 5.2 (a), $k_{1}<k_{3}$. Hence, by Lemma 5.2 (b), for all $y \in X, i_{1} \succ_{y} i_{3}$ and no cycles exist.

Step 4: "(i) and (ii) $\Rightarrow$ priority-togetherness"

Let $\mathcal{S}$ satisfy (i) and (ii). Then, Lemma 5.2 applies as well.

Assume that priority-togetherness is violated, i.e., there exists a couple $c=$ $(i, j) \in C, m \in N$, and $x \in X$ such that either $i \succ_{x} m \succ_{x} j$ or $j \succ_{x} m \succ_{x} i$. Assume that $i \succ_{x} m \succ_{x} j$ (for $j \succ_{x} m \succ_{x} i$ interchange the roles of $i$ and $j$ ). Let $i \in S_{k}$ and $j \in S_{k^{\prime}}$. Lemma 5.2 (a) and $i \succ_{x} m \succ_{x} j$ imply that $k<k^{\prime}$. Hence, neither (ii.a) 
nor (ii.b) can be true. Thus, by (ii.c), $k^{\prime}=k+1$. Hence, $S_{k}=\{i\}$ and $S_{k+1}=\{j\}$. Let $l$ be such that $m \in S_{l}$. Since $i \succ_{x} m \succ_{x} j$, from Lemma 5.2 (b) it follows that $k<l$ and $l<k+1$, a contradiction.

Proof of Theorem 5.2 Recall that all quotas equal one. Since $\varphi^{\mathcal{S}}$ is fair and efficient (Theorem 5.1) it suffices to show uniqueness. So, suppose that $\varphi$ is fair and efficient. Let $R \in \mathcal{R}$. We prove that $\varphi(R)=\varphi^{\mathcal{S}}(R)$. Let $k \in\{1, \ldots, p\}$ be such that Step $k$ is well-defined in Definition 5.3. Assume that for all $i \in \bigcup_{l<k} S_{l}$, $\varphi_{i}(R)=\varphi_{i}^{\mathcal{S}}(R)$ (this is vacuously true for $\left.\bigcup_{l<1} S_{l} \equiv \emptyset\right)$. We prove that for any student $i \in N$ that is assigned his/her allotment $\varphi_{i}^{\mathcal{S}}(R)$ in Step $k$ of Definition 5.3, it holds that $\varphi_{i}(R)=\varphi_{i}^{\mathcal{S}}(R)$. We distinguish among the following cases:

(a) $S_{k}=\{s\}$ and $s \in S$;

(b) $S_{k}=\{s\}, s \notin S$, and $S_{k} \cup S_{k+1}=\left\{s, s^{\prime}\right\}$ such that $c=\left(s, s^{\prime}\right) \in C$ or $c=\left(s^{\prime}, s\right) \in C$;

(c) $S_{k}=\left\{s, s^{\prime}\right\}, s, s^{\prime} \in S$, and

(c.1) $\operatorname{top}\left(R_{s}, X_{k}\right) \neq \operatorname{top}\left(R_{S^{\prime}}, X_{k}\right)$ or $\operatorname{top}\left(R_{S}, X_{k}\right)=\operatorname{top}\left(R_{S^{\prime}}, X_{k}\right)=0$;

(c.2) top $\left(R_{s}, X_{k}\right)=\operatorname{top}\left(R_{s^{\prime}}, X_{k}\right) \equiv \hat{x} \in X$ and $s \succ_{\hat{x}} s^{\prime}$;

(c.3) $\operatorname{top}\left(R_{s}, X_{k}\right)=\operatorname{top}\left(R_{s^{\prime}}, X_{k}\right) \equiv \hat{x} \in X$ and $s^{\prime} \succ_{\hat{x}} s$;

(d) $S_{k}=\left\{s, s^{\prime}\right\}$ and $c=\left(s, s^{\prime}\right) \in C$ or $c=\left(s^{\prime}, s\right) \in C$.

Given allocation $\varphi(R), x \in X \cup\{0\}$, and $s \in N,\left(\varphi_{-s}(R), x\right)$ denotes the allocation obtained from $\varphi(R)$ by replacing $\varphi_{s}(R)$ by $x$, whenever this is feasible.

(a) Let $\hat{x} \equiv \operatorname{top}\left(R_{s}, X_{k}\right)=\varphi_{s}^{\mathcal{S}}(R)$. Assume $\varphi_{s}(R) \neq \hat{x}$. If $\hat{x}=0$ or $[\hat{x} \in X$ and there is no $i \in N$ with $\varphi_{i}(R)=\hat{x}$ ], then allocation $\alpha \equiv\left(\varphi_{-i}(R), \hat{x}\right)$ Pareto dominates $\varphi(R)$, i.e., $\alpha_{s} P_{s} \varphi_{s}(R)$ and for all $p \in(S \cup C) \backslash\{s\}, \alpha_{p} R_{p} \varphi_{p}(R)$, contradicting efficiency. So, $\hat{x} \in X$ and there is $i \in N \backslash\{s\}$ with $\varphi_{i}(R)=\hat{x}$. By the induction hypothesis, $i \in N \backslash \bigcup_{l=1}^{k} S_{l}$. By Lemma 5.2 (b), $s \succ_{\hat{x}} i$. Since $\varphi_{S}^{\mathcal{S}}(R)=\hat{x} P_{S} \varphi_{s}(R)$, allocation $\varphi(R)$ violates the priority of $s$ (D3.2); a contradiction. Hence, $\varphi_{s}(R)=\varphi_{s}^{\mathcal{S}}(R)$.

Given allocation $\varphi(R), x, y \in X \cup\{0\}$, and $c \in C,\left(\varphi_{-c}(R),(x, y)\right)$ denotes the allocation obtained from $\varphi(R)$ by replacing $\varphi_{c}(R)$ by $(x, y)$, whenever this is feasible.

(b) Assume without loss of generality that $c=\left(s, s^{\prime}\right) \in C$. Let $(\hat{x}, \hat{y})=$ $\operatorname{top}\left(R_{c}, X_{k}\right)=\varphi_{c}^{\mathcal{S}}(R)$. Assume $\varphi_{c}(R) \neq(\hat{x}, \hat{y})$.

Suppose $\varphi_{c}(R)=(\hat{y}, \hat{x})$. Then, allocation $\alpha \equiv\left(\varphi_{-c}(R),(\hat{x}, \hat{y})\right)$ Pareto dominates $\varphi(R)$, i.e., $\alpha_{c} P_{c} \varphi_{c}(R)$ and for all $p \in(S \cup C) \backslash\{c\}, \alpha_{p} R_{p} \varphi_{p}(R)$, contradicting efficiency.

Suppose $\varphi_{c}(R)=(x, \hat{y})$ for some $x \neq \hat{x}$. If $\hat{x}=0$ or $[\hat{x} \in X$ and there is no $i \in N$ with $\left.\varphi_{i}(R)=\hat{x}\right]$, then allocation $\left(\varphi_{-c}(R),(\hat{x}, \hat{y})\right)$ Pareto dominates $\varphi(R)$, contradicting efficiency. So, $\hat{x} \in X$ and there is $i \in N \backslash\left\{s, s^{\prime}\right\}$ with $\varphi_{i}(R)=\hat{x}$. By the induction hypothesis, $i \in N \backslash \bigcup_{l=1}^{k+1} S_{l}$. By Lemma 5.2 (b), $s \succ_{\hat{x}} i$. Since $(\hat{x}, \hat{y}) P_{c}(x, \hat{y})$, or equivalently $\varphi_{c}^{\mathcal{S}}(R) P_{c} \varphi_{c}(R)$, allocation $\varphi(R)$ violates the priority of $c$ (D3.3, a.1); a contradiction.

Suppose $\varphi_{c}(R)=(\hat{x}, y)$ for some $y \neq \hat{y}$. If $\hat{y}=0$ or $[\hat{y} \in X$ and there is no $i \in N$ with $\left.\varphi_{i}(R)=\hat{y}\right]$, then allocation $\left(\varphi_{-c}(R),(\hat{x}, \hat{y})\right)$ Pareto dominates $\varphi(R)$, contradicting efficiency. So, $\hat{y} \in X$ and there is $i \in N \backslash\left\{s, s^{\prime}\right\}$ with $\varphi_{i}(R)=\hat{y}$. 
By the induction hypothesis, $i \in N \backslash \bigcup_{l=1}^{k+1} S_{l}$. By Lemma $5.2(\mathrm{~b}), s^{\prime} \succ_{\hat{y}} i$. Since $(\hat{x}, \hat{y}) P_{c}(\hat{x}, y)$, or equivalently $\varphi_{c}^{\mathcal{S}}(R) P_{c} \varphi_{c}(R)$, allocation $\varphi(R)$ violates the priority of $c$ (D3.3, a.2); a contradiction.

Suppose $\varphi_{c}(R)=(x, \hat{x})$ for some $x \neq \hat{y}$. If $\hat{y}=0$ or $[\hat{y} \in X$ and there is no $i \in N$ with $\left.\varphi_{i}(R)=\hat{y}\right]$, then allocation $\left(\varphi_{-c}(R),(\hat{x}, \hat{y})\right)$ Pareto dominates $\varphi(R)$, contradicting efficiency. So, $\hat{y} \in X$ and there is $i \in N \backslash\left\{s, s^{\prime}\right\}$ with $\varphi_{i}(R)=\hat{y}$. By the induction hypothesis, $i \in N \backslash \bigcup_{l=1}^{k+1} S_{l}$. By Lemma $5.2(\mathrm{~b}), s^{\prime} \succ_{\hat{y}} i$. If $\hat{x}=0$, then since $(\hat{x}, \hat{y}) P_{c}(x, \hat{x})$, or equivalently $\varphi_{c}^{\mathcal{S}}(R) P_{c} \varphi_{c}(R)$, allocation $\varphi(R)$ violates the priority of $c$ (D3.3, a.2); a contradiction. If $\hat{x} \in X$, then since $S_{k}=\{s\}$ and $S_{k+1}=\left\{s^{\prime}\right\}, s \succ_{\hat{x}} s^{\prime}$. Thus, since $(\hat{x}, \hat{y}) P_{c}(x, \hat{x})$, or equivalently $\varphi_{c}^{\mathcal{S}}(R) P_{c} \varphi_{c}(R)$, allocation $\varphi(R)$ violates the priority of $c$ (D3.3, c); a contradiction.

Suppose $\varphi_{c}(R)=(\hat{y}, y)$ for some $y \neq \hat{x}$. If $\hat{x}=0$ or $[\hat{x} \in X$ and there is no $i \in N$ with $\left.\varphi_{i}(R)=\hat{x}\right]$, then allocation $\left(\varphi_{-c}(R),(\hat{x}, \hat{y})\right)$ Pareto dominates $\varphi(R)$, contradicting efficiency. So, $\hat{x} \in X$ and there is $i \in N \backslash\left\{s, s^{\prime}\right\}$ with $\varphi_{i}(R)=\hat{x}$. By the induction hypothesis, $i \in N \backslash \bigcup_{l=1}^{k+1} S_{l}$. By Lemma 5.2 (b), $s \succ_{\hat{x}} i$. If $\hat{y}=0$, then since $(\hat{x}, \hat{y}) P_{c}(\hat{y}, y)$, or equivalently $\varphi_{c}^{\mathcal{S}}(R) P_{c} \varphi_{c}(R)$, allocation $\varphi(R)$ violates the priority of $c$ (D3.3, a.1); a contradiction. If $\hat{y} \in X$, then since $(\hat{x}, \hat{y}) P_{c}(\hat{y}, y)$, or equivalently $\varphi_{c}^{\mathcal{S}}(R) P_{c} \varphi_{c}(R)$, allocation $\varphi(R)$ violates the priority of $c$ after reallocation (D5.4, r.1); a contradiction.

Finally, suppose $\varphi_{c}(R)=(x, y)$ for some $x, y \notin\{\hat{x}, \hat{y}\}$.

If $\left(\hat{x}=0\right.$ or $\left[\hat{x} \in X\right.$ and there is no $i \in N \backslash\left\{s, s^{\prime}\right\}$ with $\left.\left.\varphi_{i}(R)=\hat{x}\right]\right)$ and $\left(\hat{y}=0\right.$ or $\left[\hat{y} \in X\right.$ and there is no $i \in N \backslash\left\{s, s^{\prime}\right\}$ with $\left.\left.\varphi_{i}(R)=\hat{y}\right]\right)$, then allocation $\left(\varphi_{-c}(R),(\hat{x}, \hat{y})\right)$ Pareto dominates $\varphi(R)$, contradicting efficiency. So, there is $i \in N \backslash\left\{s, s^{\prime}\right\}$ such that $\left[\hat{x} \in X\right.$ and $\left.\varphi_{i}(R)=\hat{x}\right]$ or $\left[\hat{y} \in X\right.$ and $\left.\varphi_{i}(R)=\hat{y}\right]$. By the induction hypothesis, $i \in N \backslash \bigcup_{l=1}^{k+1} S_{l}$. By Lemma 5.2 (b), [if $\hat{x} \in X$ and $\varphi_{i}(R)=\hat{x}$, then $s \succ_{\hat{x}} i$ ] and [if $\hat{y} \in X$ and $\varphi_{i}(R)=\hat{y}$, then $s^{\prime} \succ_{\hat{y}} i$ ].

If there is $i \in N \backslash\left\{s, s^{\prime}\right\}$ such that $\left[\hat{x} \in X\right.$ and $\left.\varphi_{i}(R)=\hat{x}\right]$ and $\hat{y}=0$, then, since $(\hat{x}, \hat{y}) P_{c}(x, y)$, or equivalently $\varphi_{c}^{\mathcal{S}}(R) P_{c} \varphi_{c}(R)$, allocation $\varphi(R)$ violates the priority of $c$ (D3.3, a.1); a contradiction.

If there is $i \in N \backslash\left\{s, s^{\prime}\right\}$ such that $\left[\hat{y} \in X\right.$ and $\left.\varphi_{i}(R)=\hat{y}\right]$ and $\hat{x}=0$, then, since $(\hat{x}, \hat{y}) P_{c}(x, y)$, or equivalently $\varphi_{c}^{\mathcal{S}}(R) P_{c} \varphi_{c}(R)$, allocation $\varphi(R)$ violates the priority of $c$ (D3.3, a.2); a contradiction.

If there is $i \in N \backslash\left\{s, s^{\prime}\right\}$ such that $\left[\hat{x} \in X\right.$ and $\left.\varphi_{i}(R)=\hat{x}\right]$ and $\hat{y} \in X \backslash\left\{\varphi_{l}(R): l \in\right.$ $N\}$, then, since $(\hat{x}, \hat{y}) P_{c}(x, y)$, or equivalently $\varphi_{c}^{\mathcal{S}}(R) P_{c} \varphi_{c}(R)$, allocation $\varphi(R)$ violates the priority of $c$ taking into account vacancies (D5.5, v.1); a contradiction.

If there is $i \in N \backslash\left\{s, s^{\prime}\right\}$ such that $\left[\hat{y} \in X\right.$ and $\left.\varphi_{i}(R)=\hat{y}\right]$ and $\hat{x} \in X \backslash\left\{\varphi_{l}(R): l \in\right.$ $N\}$, then, since $(\hat{x}, \hat{y}) P_{c}(x, y)$, or equivalently $\varphi_{c}^{\mathcal{S}}(R) P_{c} \varphi_{c}(R)$, allocation $\varphi(R)$ violates the priority of $c$ taking into account vacancies (D5.5, v.2); a contradiction.

If there are $i, j \in N \backslash\left\{s, s^{\prime}\right\}$ such that $\left[\hat{x} \in X\right.$ and $\left.\varphi_{i}(R)=\hat{x}\right]$ and $[\hat{y} \in X$ and $\left.\varphi_{j}(R)=\hat{y}\right]$, then $s \succ_{\hat{x}} i$ and $s^{\prime} \succ_{\hat{y}} j$. Thus, since $(\hat{x}, \hat{y}) P_{c}(x, y)$, or equivalently $\varphi_{c}^{\mathcal{S}}(R) P_{c} \varphi_{c}(R)$, allocation $\varphi(R)$ violates the priority of $c$ (D3.3, c); a contradiction.

Hence, to summarize Case (b), $\varphi_{c}(R)=\varphi_{c}^{\mathcal{S}}(R)$. 
Given allocation $\varphi(R), x, y \in X \cup\{0\}$, and $i, j \in N,\left(\varphi_{-i, j}(R), x, y\right)$ denotes the allocation obtained from $\varphi(R)$ by replacing $\varphi_{i}(R)$ with $x$ and $\varphi_{j}(R)$ with $y$, whenever this is feasible.

(c.1) Let $\hat{x}=\operatorname{top}\left(R_{s}, X_{k}\right)$ and $\hat{y}=\operatorname{top}\left(R_{s^{\prime}}, X_{k}\right)$. If $\hat{x}=\hat{y}=0$ and $\left[\varphi_{s}(R) \neq 0\right.$ or $\varphi_{s^{\prime}}(R) \neq 0$ ], then efficiency is violated. Hence we can assume that $\hat{x} \neq \hat{y}$ and $[\hat{x} \neq 0$ or $\hat{y} \neq 0]$.

Assume $\varphi_{s}(R) \neq \hat{x}$ and $\varphi_{s^{\prime}}(R) \neq \hat{x}$. If $\hat{x}=0$ or $[\hat{x} \in X$ and there is no $i \in N$ with $\left.\varphi_{i}(R)=\hat{x}\right]$, then allocation $\left(\varphi_{-s}(R), \hat{x}\right)$ Pareto dominates $\varphi(R)$, contradicting efficiency. So $\hat{x} \in X$ and there is $i \in N \backslash\left\{s, s^{\prime}\right\}$ with $\varphi_{i}(R)=\hat{x}$. By the induction hypothesis, $i \in N \backslash \bigcup_{l=1}^{k} S_{l}$. By Lemma 5.2 (b), $s \succ_{\hat{x}} i$. Since $\hat{x} P_{s} \varphi_{s}(R)$, allocation $\varphi(R)$ violates the priority of $s$ (D3.2); a contradiction. Hence, $\varphi_{s}(R)=\hat{x}$ or $\varphi_{s^{\prime}}(R)=\hat{x}$. Similarly, $\varphi_{s}(R)=\hat{y}$ or $\varphi_{s^{\prime}}(R)=\hat{y}$.

Suppose $\varphi_{s}(R)=\hat{y}$ and $\varphi_{s^{\prime}}(R)=\hat{x}$. Then, allocation $\left(\varphi_{-s, s^{\prime}}(R), \hat{x}, \hat{y}\right)$ Pareto dominates $\varphi(R)$, contradicting efficiency. So, $\varphi_{S}(R)=\hat{x}=\varphi_{s}^{\mathcal{S}}(R)$ and $\varphi_{s^{\prime}}(R)=$ $\hat{y}=\varphi_{S^{\prime}}^{\mathcal{S}}(R)$.

(c.2) Recall $\hat{x}=\operatorname{top}\left(R_{s}, X_{k}\right)$. Let $\hat{y}=\operatorname{top}\left(R_{s^{\prime}}, X_{k} \backslash\{\hat{x}\}\right)$. Note $\hat{x} \neq \hat{y}$.

Assume $\varphi_{s}(R) \neq \hat{x}$ and $\varphi_{s^{\prime}}(R) \neq \hat{x}$. If $\hat{x}=0$ or $[\hat{x} \in X$ and there is no $i \in N$ with $\left.\varphi_{i}(R)=\hat{x}\right]$, then allocation $\left(\varphi_{-s}(R), \hat{x}\right)$ Pareto dominates $\varphi(R)$, contradicting efficiency. So, $\hat{x} \in X$ and there is $i \in N \backslash\left\{s, s^{\prime}\right\}$ with $\varphi_{i}(R)=\hat{x}$. By Lemma 5.2 (b), $s \succ_{\hat{x}} i$. Since $\hat{x} P_{s} \varphi_{s}(R)$, allocation $\varphi(R)$ violates the priority of $s$ (D3.2); a contradiction. Hence, either $\varphi_{s}(R)=\hat{x}$ or $\varphi_{s^{\prime}}(R)=\hat{x}$. Similarly, either $\varphi_{s}(R)=\hat{y}$ or $\varphi_{s^{\prime}}(R)=\hat{y}$.

Suppose $\varphi_{s}(R)=\hat{y}$ and $\varphi_{s^{\prime}}(R)=\hat{x}$. Then, $s \succ_{\hat{x}} s^{\prime}$ and $\hat{x} P_{s} \hat{y}$ imply that $\varphi(R)$ violates the priority of $s(\mathrm{D} 3.2)$; a contradiction. Hence, $\varphi_{S}(R)=\hat{x}=\varphi_{S}^{\mathcal{S}}(R)$ and $\varphi_{s^{\prime}}(R)=\hat{y}=\varphi_{s^{\prime}}^{\mathcal{S}}(R)$.

(c.3) An argument similar as in (c.2) shows that $\varphi_{S}(R)=\varphi_{S}^{\mathcal{S}}(R)$ and $\varphi_{s^{\prime}}(R)=$ $\varphi_{\mathbf{s}^{\prime}}^{\mathcal{S}}(R)$.

(d) Assume without loss of generality that $c=\left(s, s^{\prime}\right) \in C$. Let $(\hat{x}, \hat{y})=$ $\operatorname{top}\left(R_{c}, X_{k}\right)=\varphi_{c}^{\mathcal{S}}(R)$. Assume $\varphi_{c}(R) \neq(\hat{x}, \hat{y})$.

Suppose $\varphi_{c}(R)=(\hat{y}, \hat{x})$. Then, allocation $\left(\varphi_{-c}(R),(\hat{x}, \hat{y})\right)$ Pareto dominates $\varphi(R)$, contradicting efficiency.

Suppose $\varphi_{c}(R)=(x, \hat{y})$ for some $x \neq \hat{x}$. If $\hat{x}=0$ or $[\hat{x} \in X$ and there is no $i \in N$ with $\left.\varphi_{i}(R)=\hat{x}\right]$, then allocation $\left(\varphi_{-c}(R),(\hat{x}, \hat{y})\right)$ Pareto dominates $\varphi(R)$, contradicting efficiency. So, $\hat{x} \in X$ and there is $i \in N \backslash\left\{s, s^{\prime}\right\}$ with $\varphi_{i}(R)=\hat{x}$. By the induction hypothesis, $i \in N \backslash \bigcup_{l=1}^{k} S_{l}$. By Lemma 5.2 (b), $s \succ_{\hat{x}} i$. Since $(\hat{x}, \hat{y}) P_{c}(x, \hat{y})$, or equivalently $\varphi_{c}^{\mathcal{S}}(R) P_{c} \varphi_{c}(R)$, allocation $\varphi(R)$ violates the priority of $c(\mathrm{D} 3.3$, a.1); a contradiction.

Suppose $\varphi_{c}(R)=(\hat{x}, y)$ for some $y \neq \hat{y}$. If $\hat{y}=0$ or $[\hat{y} \in X$ and there is no $i \in N$ with $\left.\varphi_{i}(R)=\hat{y}\right]$, then allocation $\left(\varphi_{-c}(R),(\hat{x}, \hat{y})\right)$ Pareto dominates $\varphi(R)$, contradicting efficiency. So, $\hat{y} \in X$ and there is $i \in N \backslash\left\{s, s^{\prime}\right\}$ with $\varphi_{i}(R)=\hat{y}$. By the induction hypothesis, $i \in N \backslash \bigcup_{l=1}^{k} S_{l}$. By Lemma 5.2 (b), $s^{\prime} \succ_{\hat{y}} i$. Since $(\hat{x}, \hat{y}) P_{c}(\hat{x}, y)$, or equivalently $\varphi_{c}^{\mathcal{S}}(R) P_{c} \varphi_{c}(R)$, allocation $\varphi(R)$ violates the priority of $c(\mathrm{D} 3.3$, a.2); a contradiction.

Suppose $\varphi_{c}(R)=(x, \hat{x})$ for some $x \neq \hat{y}$. If $\hat{y}=0$ or $[\hat{y} \in X$ and there is no $i \in N$ with $\left.\varphi_{i}(R)=\hat{y}\right]$, then allocation $\left(\varphi_{-c}(R),(\hat{x}, \hat{y})\right)$ Pareto dominates $\varphi(R)$, 
contradicting efficiency. So, $\hat{y} \in X$ and there is $i \in N \backslash\left\{s, s^{\prime}\right\}$ with $\varphi_{i}(R)=\hat{y}$. By the induction hypothesis, $i \in N \backslash \bigcup_{l=1}^{k} S_{l}$. By Lemma $5.2(\mathrm{~b}), s^{\prime} \succ_{\hat{y}} i$. If $\hat{x}=0$, then since $(\hat{x}, \hat{y}) P_{c}(x, \hat{x})$, or equivalently $\varphi_{c}^{\mathcal{S}}(R) P_{c} \varphi_{c}(R)$, allocation $\varphi(R)$ violates the priority of $c$ (D3.3, a.2); a contradiction. If $\hat{x} \in X$, then since $(\hat{x}, \hat{y}) P_{c}(x, \hat{x})$, or equivalently $\varphi_{c}^{\mathcal{S}}(R) P_{c} \varphi_{c}(R)$, allocation $\varphi(R)$ violates the priority of $c$ after reallocation (D5.4, r.2); a contradiction.

Suppose $\varphi_{c}(R)=(\hat{y}, y)$ for some $y \neq \hat{x}$. If $\hat{x}=0$ or $[\hat{x} \in X$ and there is no $i \in N$ with $\left.\varphi_{i}(R)=\hat{x}\right]$, then allocation $\left(\varphi_{-c}(R),(\hat{x}, \hat{y})\right)$ Pareto dominates $\varphi(R)$, contradicting efficiency. So, $\hat{x} \in X$ and there is $i \in N \backslash\left\{s, s^{\prime}\right\}$ with $\varphi_{i}(R)=\hat{x}$. By the induction hypothesis, $i \in N \backslash \bigcup_{l=1}^{k} S_{l}$. By Lemma 5.2 (b), $s \succ_{\hat{x}} i$. If $\hat{y}=0$, then since $(\hat{x}, \hat{y}) P_{c}(\hat{y}, y)$, or equivalently $\varphi_{c}^{\mathcal{S}}(R) P_{c} \varphi_{c}(R)$, allocation $\varphi(R)$ violates the priority of $c$ (D3.3, a.1); a contradiction. If $\hat{y} \in X$, then since $(\hat{x}, \hat{y}) P_{c}(\hat{y}, y)$, or equivalently $\varphi_{c}^{\mathcal{S}}(R) P_{c} \varphi_{c}(R)$, allocation $\varphi(R)$ violates the priority of $c$ after reallocation (D5.4, r.1); a contradiction.

Finally, suppose $\varphi_{c}(R)=(x, y)$ for some $x, y \notin\{\hat{x}, \hat{y}\}$.

If $\left(\hat{x}=0\right.$ or $\left[\hat{x} \in X\right.$ and there is no $i \in N \backslash\left\{s, s^{\prime}\right\}$ with $\left.\left.\varphi_{i}(R)=\hat{x}\right]\right)$ and $\left(\hat{y}=0\right.$ or $\left[\hat{y} \in X\right.$ and there is no $i \in N \backslash\left\{s, s^{\prime}\right\}$ with $\left.\left.\varphi_{i}(R)=\hat{y}\right]\right)$, then allocation $\left(\varphi_{-c}(R),(\hat{x}, \hat{y})\right)$ Pareto dominates $\varphi(R)$, contradicting efficiency. So, there is $i \in N \backslash\left\{s, s^{\prime}\right\}$ such that $\left[\hat{x} \in X\right.$ and $\left.\varphi_{i}(R)=\hat{x}\right]$ or $\left[\hat{y} \in X\right.$ and $\left.\varphi_{i}(R)=\hat{y}\right]$. By the induction hypothesis, $i \in N \backslash \bigcup_{l=1}^{k} S_{l}$. By Lemma 5.2 (b), [if $\hat{x} \in X$ and $\varphi_{i}(R)=\hat{x}$, then $s \succ_{\hat{x}} i$ ] and [if $\hat{y} \in X$ and $\varphi_{i}(R)=\hat{y}$, then $s^{\prime} \succ_{\hat{y}} i$ ].

If there is $i \in N \backslash\left\{s, s^{\prime}\right\}$ such that $\left[\hat{x} \in X\right.$ and $\left.\varphi_{i}(R)=\hat{x}\right]$ and $\hat{y}=0$, then, since $(\hat{x}, \hat{y}) P_{c}(x, y)$, or equivalently $\varphi_{c}^{\mathcal{S}}(R) P_{c} \varphi_{c}(R)$, allocation $\varphi(R)$ violates the priority of $c$ (D3.3, a.1); a contradiction.

If there is $i \in N \backslash\left\{s, s^{\prime}\right\}$ such that $\left[\hat{y} \in X\right.$ and $\left.\varphi_{i}(R)=\hat{y}\right]$ and $\hat{x}=0$, then, since $(\hat{x}, \hat{y}) P_{c}(x, y)$, or equivalently $\varphi_{c}^{\mathcal{S}}(R) P_{c} \varphi_{c}(R)$, allocation $\varphi(R)$ violates the priority of $c(\mathrm{D} 3.3, \mathrm{a} .2)$; a contradiction.

If there is $i \in N \backslash\left\{s, s^{\prime}\right\}$ such that $\left[\hat{x} \in X\right.$ and $\left.\varphi_{i}(R)=\hat{x}\right]$ and $\hat{y} \in X \backslash\left\{\varphi_{l}(R): l \in\right.$ $N\}$, then, since $(\hat{x}, \hat{y}) P_{c}(x, y)$, or equivalently $\varphi_{c}^{\mathcal{S}}(R) P_{c} \varphi_{c}(R)$, allocation $\varphi(R)$ violates the priority of $c$ taking into account vacancies (D5.5, v.1); a contradiction.

If there is $i \in N \backslash\left\{s, s^{\prime}\right\}$ such that $\left[\hat{y} \in X\right.$ and $\left.\varphi_{i}(R)=\hat{y}\right]$ and $\hat{x} \in X \backslash\left\{\varphi_{l}(R): l \in\right.$ $N\}$, then, since $(\hat{x}, \hat{y}) P_{c}(x, y)$, or equivalently $\varphi_{c}^{\mathcal{S}}(R) P_{c} \varphi_{c}(R)$, allocation $\varphi(R)$ violates the priority of $c$ taking into account vacancies (D5.5, v.2); a contradiction.

If there are $i, j \in N \backslash\left\{s, s^{\prime}\right\}$ such that $\left[\hat{x} \in X\right.$ and $\left.\varphi_{i}(R)=\hat{x}\right]$ and $[\hat{y} \in X$ and $\left.\varphi_{j}(R)=\hat{y}\right]$, then $s \succ_{\hat{x}} i$ and $s^{\prime} \succ_{\hat{y}} j$. Thus, since $(\hat{x}, \hat{y}) P_{c}(x, y)$, or equivalently $\varphi_{c}^{\mathcal{S}}(R) P_{c} \varphi_{c}(R)$, allocation $\varphi(R)$ violates the priority of $c$ (D3.3, c); a contradiction.

Hence, to summarize Case $(\mathrm{d}), \varphi_{c}(R)=\varphi_{c}^{\mathcal{S}}(R)$.

Proof of Proposition 5.1 Suppose that $\varphi$ is fair and efficient, but not vacancyfair on $\mathcal{R} \mathcal{R}$. Then there is $R \in \mathcal{R} \mathcal{R}$ such that allocation $\varphi(R)$ violates the priority of $c$ taking into account vacancies (D5.5). Let $\alpha_{l}:=\varphi_{l}(R)$ for all $l \in N$. Assume, without loss of generality, that Definition 5.5 (v.1) applies. Hence, there exist 
$x \in X, k \in N \backslash\{i, j\}$, and $v \in X \backslash\left\{\alpha_{l}: l \in N\right\}$ such that $\alpha_{k}=x, i \succ_{x} k$, and $(x, v) P_{(i, j)}\left(\alpha_{i}, \alpha_{j}\right)$. By responsiveness of couple $c$ 's preferences associated individual preferences $R_{i}, R_{j} \in \mathcal{R} \mathcal{S}$ exist for students $i$ and $j$ such that $x P_{i} \alpha_{i}$ or $v P_{j} \alpha_{j}$.

If $x P_{i} \alpha_{i}$, then by responsiveness, $\left(x, \alpha_{j}\right) P_{c}\left(\alpha_{i}, \alpha_{j}\right)$; a violation of couple $c$ 's priority (D3.3, a.1). So, $v P_{j} \alpha_{j}$. But then, by responsiveness, $\left(\alpha_{i}, v\right) P_{c}\left(\alpha_{i}, \alpha_{j}\right)$, contradicting efficiency.

Proof of Theorem 5.3 Let $\succ$ be a pt-acyclic priority structure that induces the strongly $p t$-acyclic partition $\mathcal{S}=\left\{S_{1}, \ldots, S_{p}\right\}$. Let $R \in \mathcal{R}$. In each step of the sequential placement mechanism either a couple chooses its best pair of available positions or a set of students obtain efficient allotments through the deferred acceptance algorithm (because of the acyclicity the deferred acceptance algorithm allocation is an efficient allocation for the reduced placement problem, see Ergin 2002, Theorem 1). Hence, there is an order $\sigma(R)$ of the students (i.e., single students and couples) associated with the execution of the sequential placement mechanism such that $\varphi^{\mathcal{S}}(R)$ equals the allocation obtained by applying the serial dictatorship implied by $\sigma(R)$. Hence, $\varphi^{\mathcal{S}}(R)$ is efficient. ${ }^{9}$

It remains to prove that $\varphi^{\mathcal{S}}(R)$ does not violate the priority of any single student or any couple. Let $k \in\{1, \ldots, p\}$ be such that Step $k$ is well-defined in Definition 5.8. Assume that $\varphi^{\mathcal{S}}(R)$ does not violate the priority of any single student and any couple in $\bigcup_{l<k} S_{l}$ (this is vacuously true for $\bigcup_{l<1} S_{l} \equiv \emptyset$ ). We prove that $\varphi^{\mathcal{S}}(R)$ also does not violate the priority of any single student and any couple involved in Step $k$. We distinguish among the following cases:

(a) $S_{k} \subseteq S$;

(b) $S_{k}=\{s\}, s \notin S$, and $S_{k} \cup S_{k+1}=\left\{s, s^{\prime}\right\}$ such that $c=\left(s, s^{\prime}\right) \in C$ or $c=\left(s^{\prime}, s\right) \in C$;

(c) $S_{k}=\left\{s, s^{\prime}\right\}$ and $c=\left(s, s^{\prime}\right) \in C$ or $c=\left(s^{\prime}, s\right) \in C$.

(a) Let $s \in S_{k}$. By Definition 5.8, all position types that student $s$ likes better than $\varphi_{S}^{\mathcal{S}}(R)$ are assigned to students in $\bigcup_{l<k} S_{l}$ or to other students in $S_{k}$. Since all students in $\bigcup_{l<k} S_{l}$ have a higher priority for all position types (D5.6, ii), $\varphi^{\mathcal{S}}(R)$ does not violate the priority of $s$ because of any student in $\bigcup_{l<k} S_{l}$. Furthermore, since the allocation for students in $S_{k}$ is obtained by applying the DA algorithm, which always produces a fair allocation, to the reduced problem that only contains students in $S_{k}, \varphi^{\mathcal{S}}(R)$ does not violate the priority of $s$ because of any other student in $S_{k}$. Thus, $\varphi^{\mathcal{S}}(R)$ does not violate the priority of $s$.

(b) Without loss of generality assume that $c=\left(s, s^{\prime}\right) \in C$. Suppose $\varphi^{\mathcal{S}}(R)$ violates the priority of $c$. There are five subcases (see Definition 3.3) that we have to distinguish for this violation. However, we only consider the first subcase, Definition 3.3 (a.1), as the other subcases run analogously. There exist $x \in X$ and $\tilde{s} \in N \backslash\left\{s, s^{\prime}\right\}$ such that $\varphi_{\tilde{s}}^{\mathcal{S}}(R)=x, s \succ_{x} \tilde{s}$, and $\left(x, \varphi_{s^{\prime}}^{\mathcal{S}}(R)\right) P_{c}\left(\varphi_{s}^{\mathcal{S}}(R), \varphi_{s^{\prime}}^{\mathcal{S}}(R)\right)$ or $(x, 0) P_{c}\left(\varphi_{s}^{\mathcal{S}}(R), \varphi_{s^{\prime}}^{\mathcal{S}}(R)\right)$. From Definition 5.6, (ii), $s \succ_{x} \tilde{s}$, and $\tilde{s} \in N \backslash\left\{s, s^{\prime}\right\}$ it

9 In Example 5.7, for instance, $\sigma(R)=(5,6,7,(1,2),(3,4), 8)$ (in fact any sequence of students 5, 6, and 7 at the beginning of the serial dictatorship is possible for this specific example). 
follows that $\left(x, \varphi_{S^{\prime}}^{\mathcal{S}}(R)\right)$ and $(x, 0)$ were available options at Step $k$. Apparently, $c$ was not assigned its most favorite position assignment according to $q^{k}$, a contradiction to Definition 5.8. Hence, $\varphi^{\mathcal{S}}(R)$ does not violate the priority of $c$. (c) A similar argument as in (b) shows that $\varphi^{\mathcal{S}}(R)$ does not violate the priority of couple $c$.

\section{References}

Alkan A, Gale D (2003) Stable schedule matching under revealed preferences. J Econ Theory 112:289-306

Balinski M, Sönmez T (1999) A tale of two mechanisms: student placement. J Econ Theory 84:73-94

Ehlers L (2002) Coalitional strategy-proof house allocation. J Econ Theory 105:298-317

Ehlers L, Klaus B (2003) Resource-monotonic house allocation. Int J Game Theory 32:545-560

Ehlers L, Klaus B (2006a) Efficient priority rules. Games Econ Behav 55:372-384

Ehlers L, Klaus B (2006b) Consistent House Allocation. Econ Theory (in press). DOI 10.1007/s00199-005-0077-z

Ehlers L, Klaus B, Pápai S (2002) Strategy-proofness and population-monotonicity for house allocation problems. J Math Econ 83:329-339

Ergin HI (2002) Efficient resource allocation on the basis of priorities. Econometrica 70:2489-2497

Gale D, Shapley LS (1962) College admissions and the stability of marriage. Am Math Mon 69:9-15

Hatfield JW, Milgrom P (2005) Auctions, matching and the law of aggregate demand. Am Econ Rev 95:913-935

Kelso AS, Crawford VP (1982) Job matching, coalition formation, and gross substitutes. Econometrica 50:1483-1504

Kesten O (2006) On two competing mechanisms for priority-based allocation problems. J Econ Theory 127:155-171

Klaus B, Klijn F (2005) Stable matchings and preferences of couples. J Econ Theory 121:75-106

Klaus B, Klijn F (2007) Paths to Stability for Matching Markets with Couples. Games Econ Behav 58:154-171 (DOI 10.1016/j.geb.2006.03.002)

Klaus B, Klijn F, Massó J (2006) Some Things Couples always Wanted to Know about Stable Matchings (but Were Afraid to Ask). Rev Econ Des (in press). DOI 10.1007/s10058-006-0017-9

Roth AE (1984) The evolution of the labor market for medical interns and residents: a case study in game theory. J Polit Econ 92:991-1016

Roth AE (1985) The college admissions problem is not equivalent to the marriage problem. J Econ Theory $36: 277-288$

Roth AE, Sotomayor MAO (1990) Two-sided matching: a study in game-theoretic modeling and analysis. Econometric Society Monograph Series. Cambridge University Press, New York 